\title{
Synthesis and biological evaluation of heterocyclic 1,2,4-triazole scaffolds as promising pharmacological agents
}

\author{
Mukesh Kumari', Sumit Tahlan', Balasubramanian Narasimhan', Kalavathy Ramasamy²,3, Siong Meng Lim²,
} Syed Adnan Ali Shah ${ }^{2,4}$, Vasudevan Mani ${ }^{5}$ and Saloni Kakkar ${ }^{1 *}$

\begin{abstract}
Background: Triazole is an important heterocyclic moiety that occupies a unique position in heterocyclic chemistry, due to its large number of biological activities. It exists in two isomeric forms i.e. 1,2,4-triazole and 1,2,3-triazole and is used as core molecule for the design and synthesis of many medicinal compounds. 1,2,4-Triazole possess broad spectrum of therapeutically interesting drug candidates such as analgesic, antiseptic, antimicrobial, antioxidant, antiurease, anti-inflammatory, diuretics, anticancer, anticonvulsant, antidiabetic and antimigraine agents.
\end{abstract}

Methods: The structures of all synthesized compounds were characterized by physicochemical properties and spectral means (IR and NMR). The synthesized compounds were evaluated for their in vitro antimicrobial activity against Gram-positive (B. subtilis), Gram-negative (P. aeruginosa and E. coli) bacterial and fungal (C. albicans and A. niger) strains by tube dilution method using ciprofloxacin, amoxicillin and fluconazole as standards. In-vitro antioxidant and antiurease screening was done by DPPH assay and indophenol method, respectively. The in-vitro anticancer evaluation was carried out against MCF-7 and HCT116 cancer cell lines using 5-FU as standards.

Results, discussion and conclusion: The biological screening results reveal that the compounds $\mathbf{T}_{\mathbf{5}}\left(\mathrm{MIC}_{B S}\right.$, $\left.E C=24.7 \mu \mathrm{M}, \mathrm{MIC}_{P A} C_{A}=12.3 \mu \mathrm{M}\right)$ and $\mathbf{T}_{17}\left(\mathrm{MIC}_{A N}=27.1 \mu \mathrm{M}\right)$ exhibited potent antimicrobial activity as comparable to standards ciprofloxacin, amoxicillin ( $\left.\mathrm{MIC}_{\text {Cipro }}=18.1 \mu \mathrm{M}, \mathrm{MIC}_{\mathrm{Amo}}=17.1 \mu \mathrm{M}\right)$ and fluconazole $\left(\mathrm{MIC}_{\mathrm{Flu}}=20.4 \mu \mathrm{M}\right)$, respectively. The antioxidant evaluation showed that compounds $\mathbf{T}_{\mathbf{2}}\left(I \mathrm{C}_{50}=34.83 \mu \mathrm{g} / \mathrm{ml}\right)$ and $\mathbf{T}_{\mathbf{3}}\left(\mathrm{I} \mathrm{C}_{50}=34.38 \mu \mathrm{g} / \mathrm{ml}\right)$ showed significant antioxidant activity and comparable to ascorbic acid $\left(I C_{50}=35.44 \mu \mathrm{g} / \mathrm{ml}\right)$. Compounds $\mathbf{T}_{\mathbf{3}}\left(\mathrm{I} \mathrm{C}_{50}=54.01 \mu \mathrm{g} /\right.$ $\mathrm{ml}$ ) was the most potent urease inhibitor amongst the synthesized compounds and compared to standard thiourea $\left(I C_{50}=54.25 \mu \mathrm{g} / \mathrm{ml}\right)$. The most potent anticancer activity was shown by compounds $\mathbf{T}_{\mathbf{2}}\left(I C_{50}=3.84 \mu \mathrm{M}\right)$ and $\mathbf{T}_{\mathbf{7}}$ $\left(\mathrm{IC} \mathrm{C}_{50}=3.25 \mu \mathrm{M}\right)$ against $\mathrm{HCT} 116$ cell lines as compared to standard 5-FU $\left(\mathrm{IC}_{50}=25.36 \mu \mathrm{M}\right)$.

Keywords: 1,2,4-Triazole, Antimicrobial, Antioxidant, Anti-urease, Anticancer, SAR

\section{Introduction}

Triazole is an N-bridged aromatic heterocyclic compound that received a considerable attention in recent years due to their biological activities [1]. The name

\footnotetext{
*Correspondence: salonikakkar2007@gmail.com

${ }^{1}$ Faculty of Pharmaceutical Sciences, Maharshi Dayanand University, Rohtak 124001, India

Full list of author information is available at the end of the article
}

"triazole" was first use by Bladin in 1855 for describing the carbon-nitrogen ring system $\mathrm{C}_{2} \mathrm{H}_{3} \mathrm{~N}_{3}$ [2]. It is a white to pale yellow crystalline solid with a weak, characteristic odour, soluble in water and alcohol, melts at $120{ }^{\circ} \mathrm{C}$ and boils at $260{ }^{\circ} \mathrm{C}$ [3]. Triazole exists in two isomeric forms such as 1,2,4-triazole and 1,2,3-triazole [4]. The SAR studies of triazole derivative reveals that substitution on positions 3,4 and 5 of triazole ring can be varied but the greatest changed in physicochemical 
properties and biological profile is exerted by the groups attached to the nitrogen atom at the 4th position [3]. It favours the hydrogen bonding and is also stable for metabolic degradation, which could be favorable in increasing solubility as well as in binding bimolecular targets [5].
Novel triazole drugs discovered and developed by applying bioisosteric replacement technique with extending biological activities also captured a special attention in medicinal chemistry [6]. Numerous medicines containing triazole moiety available in market (Fig. 1) are: Antifungal<smiles>CCCC(C#N)(Cn1cncn1)c1ccc(Cl)cc1</smiles>

Myclobutanil (Antifungal)<smiles>CC(C)(C)C(O)(CCc1ccc(Cl)cc1)Cn1cncn1</smiles>

Tebuconazole (Antifungal)<smiles>CC(C)(C)C(O)C(Cc1ccc(Cl)cc1)n1cncn1</smiles>

Paclobutranol

(Antifungal)<smiles>[H][Z](CC)n1ncn(-c2ccc(N3CCN(c4ccc(OC[C@@H]5CO[C@@](Cn6cncn6)(c6ccc(F)cc6F)O5)cc4)CC3)cc2)c1=O</smiles>

Posaconazole Fluconazole (Antifungal) (Antifungal)

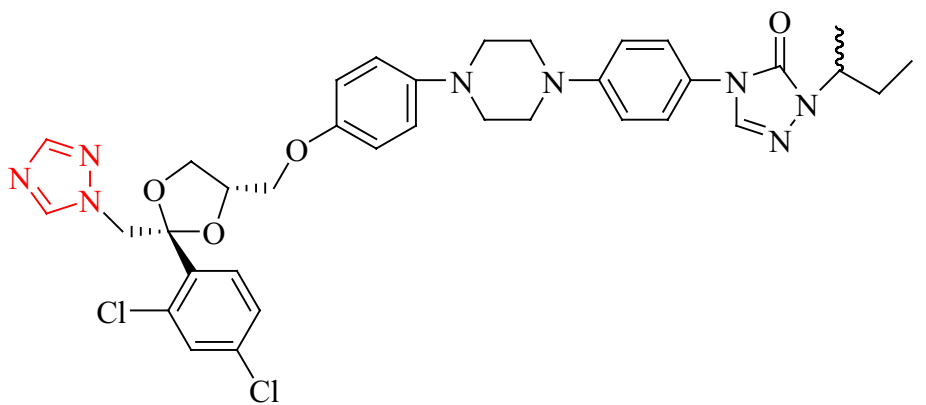

Itraconazole (Antifungal)<smiles>CC(C)(C#N)c1cc(Cn2cncn2)cc(C(C)(C)C#N)c1</smiles>

Anastrazole (Anticancer)<smiles>N=Cc1ccc(C(c2ccc(C=N)cc2)n2cncn2)cc1</smiles><smiles>Cn1nnc2ccc(C(c3ccc(Cl)cc3)n3cncn3)cc21</smiles><smiles>CN(C)CCc1c[nH]c2ccc(Cn3cncn3)cc12</smiles><smiles>NC(=O)c1ncn(C2OC3(CO)OC2C3O)n1</smiles>

\section{Litrozole Vorozole Rizatriptan Ribavirin}

(Anticancer) (Anticancer) (Antimigrain) (Antiviral)

Fig. 1 Marketed preparations containing 1,2,4-triazole as core moiety 
[7-10]-myclobutanil, tebuconazole, posaconazole, itraconazole fluconazole, paclobutrazole Anticancer [9, 11]-anastrazole, litrozole, vorozole, Antimigrain [9, 12]-rizatriptan and Antiviral [9, 13]-ribavirin.

At present time, our medical field is suffering from the problem of antimicrobial resistance towards many microbial strains. Hence as prioritized by various health organizations, there is a need for the discovery or development of novel antimicrobial compounds possessing a broad spectrum activity exhibiting high effectiveness against those highly resistant Gram positive, Gram negative bacterial and fungal strains [14].

Human cells face threats everyday because the attack of various viruses, infections and free radicals damage the body cells and DNA. Scientists observed that the free radicals contribute to the ageing process and also contribute in diseases, like cancer, diabetes and heart disease. Antioxidants are the chemicals that stop or limit the damage caused by the free radicals and also boost our immunity [15].

Ureases relate to the class of Urea amidohydrolases enzymes containing two nickel(II) atoms. Ureases are mainly obtained from plants, algae, fungi and bacteria. Bacterial ureases are responsible for causing many diseases like pyelonephritis, hepatic coma, peptic ulceration, urinary stones and stomach cancer. Rationally, A category of antiurease or urease inhibitory drugs was developed for curing the urease caused disease by inhibiting urease enzymes. The two nickel(II) atoms present in active site of Ureases accelerate the hydrolysis of urea into ammonia and carbon dioxide gas. Both $\mathrm{CO}_{2}$ and $\mathrm{NH}_{3}$ are important virulence factor for the pathogenesis of many above given clinical conditions. Anti-urease compounds inhibit the hydrolysis of urea by antagonising urease enzyme [16]. This article also focuses on some new 1,2,4-triazole derivatives exhibiting anti-urease activity.

Colorectal cancer is the third most lethal cancer worldwide in both males and females with drug resistance and metastasis being the major challenge to effective treatments. Maximum deaths due to colon cancer are related with metastatic ailment. The growth of colorectal cancer is promoted by epigenetic factors, such as abnormal DNA methylation. Targeted therapy is a kind of chemotherapy that specifically targets the proteins that resist the development of some cancers [17].

Palmitic acid (common name) is categorized as saturated fatty acid with chemical formula $\mathrm{CH}_{3}\left(\mathrm{CH}_{2}\right)_{14} \mathrm{COOH}$ (IUPAC name: hexadecanoic acid). The main sources of palmitic acid are palm oil, olive oil, meats, cheese, cocoa butter, breast milk and dairy products [18]. Napalm, is a derivative of palmitic acid, synthesized by the combination of aluminium salts of palmitic acid and naphthenic acid and it was used as fuel during World War II [19].

1,2,4-Triazole attracts the attention of researchers due to its broad spectrum of biological activities (Fig. 2) such as antimigrain [9, 12], antioxidant [15], anti-urease [16], antimicrobial [20,21], anti-inflammatory [21, 22], anticonvulsant [23], anticancer [11, 24], antiviral [25] and antiparasytic [25].

\section{Results and discussion}

\section{Chemistry}

The multistep synthetic process of 1,2,4-triazole derivatives $\left(\mathbf{T}_{\mathbf{1}}-\mathbf{T}_{\mathbf{2 0}}\right)$ was depicted in Scheme 1. Initially, ethylpalmitate (Int-i) was synthesized by the reaction of palmitic acid, ethanol and sulphuric acid. Palmitohydrazide (Int-ii) was synthesized from ethanolic solution of ethylpalmitate (Int-i) followed by addition of hydrazine hydrate. 5-Pentadecyl-1,3,4-oxadiazole2(3H)-thione (Int-iii) was synthesized using Int-ii in alc. potassium hydroxide solution followed by the addition of carbon disulfide and then followed by addition of hydrazine hydrate to Int-iii yielded 4-amino-5-pentadecyl-4H-1,2,4-triazole-3-thiol (Int-iv). Finally, the Int-iv on reaction with different substituted aromatic aldehydes in ethanol yielded the title compounds $\left(\mathbf{T}_{\mathbf{1}}-\mathbf{T}_{\mathbf{2 0}}\right)$. The physicochemical properties of the synthesized compounds are depicted in Table 1 . The synthesized derivatives of 1,2,4-triazole were confirmed by Infrared (IR) and Nuclear Magnetic Resonance $\left({ }^{1} \mathrm{H} /{ }^{13} \mathrm{CNMR}\right)$. The spectro-analytical data has been depicted in Table 2 . The presence of aliphatic - $\mathrm{CH}$ - stretch in all compounds was confirmed at $2990-2879 \mathrm{~cm}^{-1}$. The intermediates (Int-ii, iii and iv) exhibited the $-\mathrm{NH}$ stretch in range of 3424$3319 \mathrm{~cm}^{-1}$. The presence of $-\mathrm{CONH}$ - group in Int-ii was indicated by appearance of $-\mathrm{CONH}$ - stretch at $1630 \mathrm{~cm}^{-1}$. The peak range $1677-1589 \mathrm{~cm}^{-1}$ in Int-iii, iv and compounds $\mathbf{T}_{\mathbf{1}}-\mathbf{T}_{\mathbf{2 0}}$ indicated the presence of $-\mathrm{C}=\mathrm{N}$ stretch. The presence of $-\mathrm{SH}$ stretching vibrations in Intiv and compounds $\mathbf{T}_{\mathbf{1}}-\mathbf{T}_{\mathbf{2 0}}$ were indicated in a scale of 2593-2505 $\mathrm{cm}^{-1}$. The compounds $\mathbf{T}_{4}, \mathbf{T}_{5}$ and $\mathbf{T}_{6}$ showed the $-\mathrm{OCH}_{3}$ stretching vibrations in the range of 2860$2848 \mathrm{~cm}^{-1}$. The presence of phenolic group in compounds $\mathbf{T}_{6}, \mathbf{T}_{7}, \mathbf{T}_{8}$ and $\mathbf{T}_{18}$ was indicated by peaks in the range of $3483-3400 \mathrm{~cm}^{-1}$. The peak range $701-699 \mathrm{~cm}^{-1}$ of compounds $\mathbf{T}_{13}$ and $\mathbf{T}_{14}$ was indicated the presence of Ar- $\mathrm{Br}$ group. The compounds $\mathbf{T}_{15}, \mathbf{T}_{16}$ and $\mathbf{T}_{\mathbf{1 7}}$ showed the $\mathrm{Ar}-\mathrm{NO}_{2}$ stretching vibrations in the range of 1545$1424 \mathrm{~cm}^{-1}$. The presence of $\mathrm{Ar}-\mathrm{Cl}$ group in compounds $\mathbf{T}_{10}, \mathbf{T}_{11}$ and $\mathbf{T}_{12}$ was confirmed by the appearance of peaks in the range of $767-750 \mathrm{~cm}^{-1}$. The presence of tertiary amine in compound $\mathbf{T}_{\mathbf{9}}$ was confirmed by the appearance of peak at $3431 \mathrm{~cm}^{-1}$. The presence of aromatic ring in compounds $\mathbf{T}_{\mathbf{1}}-\mathbf{T}_{\mathbf{2 0}}$ was indicated by the 


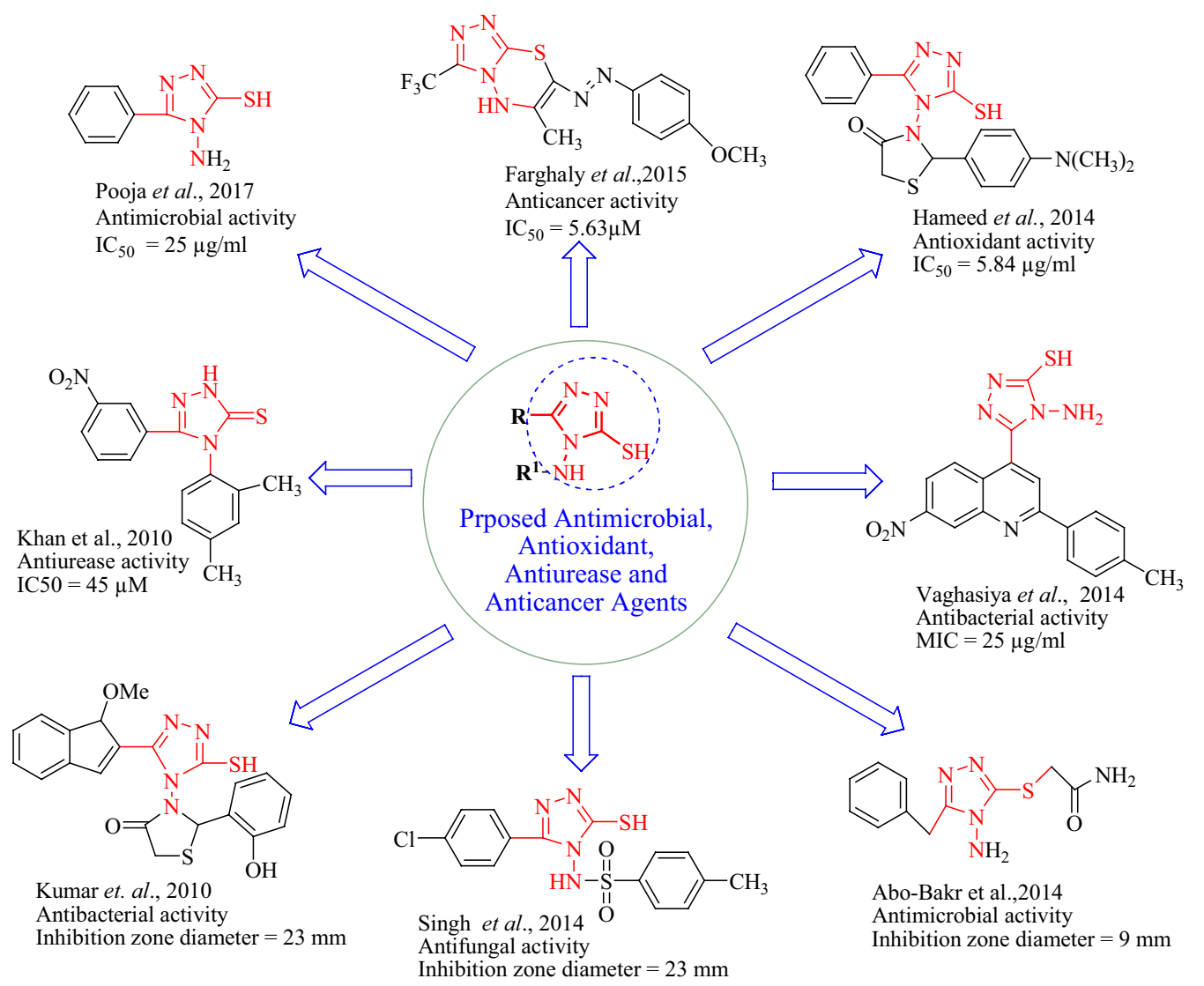

Fig. 2 Design of 1,2,4-triazole analogues for antimicrobial, antioxidant, antiurease and anticancer activities based on biological profile

appearance of peak in the range of $1796-1719 \mathrm{~cm}^{-1}$. DMSO was used as solvent for the analysis of compounds by ${ }^{1} \mathrm{HNMR}$ spectra. The presence of singlet signal at $1.22-2.47 \delta \mathrm{ppm}$ and $0.82-0.84 \delta \mathrm{ppm}$ indicated the presence of protons of $-\mathrm{CH}_{2}$ and $-\mathrm{CH}_{3}$ groups in Intii, iii and iv, respectively. Singlet at $2.25 \delta \mathrm{ppm}$ and 8.87 $\delta$ ppm showed the presence of protons of $\mathrm{NH}_{2}$ and $\mathrm{NH}$ groups in Int-ii, iii and iv, respectively. The presence of proton of SH group was indicated by appearance of singlet at 3.30 in Int-iv. The findings of elemental analysis of synthesized derivatives were recorded within theoretical results of $\pm 0.4 \%$. Mass spectra of the synthesized derivatives reflected the characteristic molecular ion peaks.

\section{Structure activity relationship (SAR) studies}

In the synthesized compounds, the substitution on $m$ - and $p$-position of the aromatic ring with methoxy group improved the antimicrobial activity (compound $\left.\mathbf{T}_{5}, \mathrm{MIC}_{B S, E C}=24.7 \mu \mathrm{M}, \mathrm{MIC}_{P A, C A}=12.3 \mu \mathrm{M}\right)$ against Gram positive (B. subtilis, P. aeruginosa), Gram negative ( $E$. coli) bacterial and fungal (C. albicans) strains, respectively. The $p$-substitution of nitro (compound $\mathbf{T}_{17}$, $\left.\mathrm{MIC}_{A N}=27.1 \mu \mathrm{M}\right)$ group improved the antifungal activity against $A$. niger. The substituent methyl at $p$-position of ring (compound $\mathbf{T}_{3}, \mathrm{IC}_{50}=54.01 \mu \mathrm{g} / \mathrm{ml}$ ) enhanced the anti-urease activity. The antioxidant activity has been improved by $p$-substituents i.e. aldehyde (compound $\mathbf{T}_{2}$, $\mathrm{IC}_{50}=34.83 \mu \mathrm{g} / \mathrm{ml}$ ) and methyl groups (compound $\mathbf{T}_{3}$, $\mathrm{IC}_{50}=34.38 \mu \mathrm{g} / \mathrm{ml}$ ). The most potent anticancer activity showed by compounds $\mathbf{T}_{2}\left(\mathrm{IC}_{50}=3.84 \mu \mathrm{M}\right)$ and $\mathbf{T}_{7}$ $\left(\mathrm{IC}_{50}=3.25 \mu \mathrm{M}\right)$ against HCT116 cell lines as compared to standard 5-FU $\left(\mathrm{IC}_{50}=25.36 \mu \mathrm{M}\right)$. From the analysis of antimicrobial activity, it may be concluded that the substitution of methoxy group increase the antibacterial activity whereas introduction of nitro as electron withdrawing groups at $p$-position may enhance the antifungal activity of synthesized compounds. The introduction of methyl substituent as electron donating groups at $p$-position of aromatic ring may increase the anti-urease as well as antioxidant activity. The substitution of of $p$-aldehyde and $o$-hydroxy group on the aromatic ring may enhance the anticancer activity against HCT116 cells (Fig. 3). 


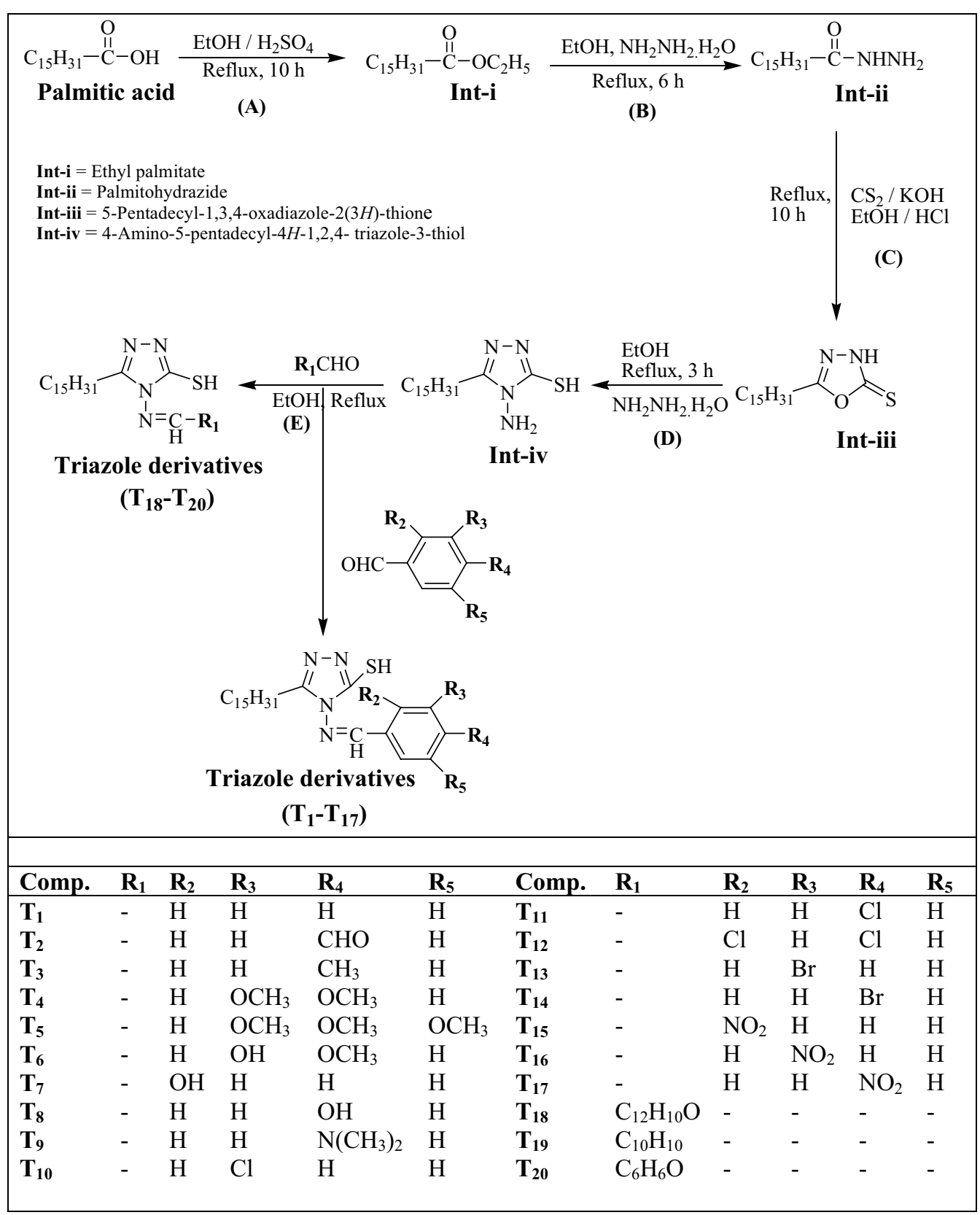

Scheme 1. Synthesis of 1,2,4-triazole derivatives 
Table 1 Physicochemical characterization of synthesized derivatives $\left(T_{1}-T_{20}\right)$

\begin{tabular}{|c|c|c|c|c|c|c|}
\hline Comp & Mol. formula & Mol. wt & Colour & M.p. $\left({ }^{\circ} \mathrm{C}\right)$ & Rf value & $\%$ Yield \\
\hline Int-ii & $\mathrm{C}_{18} \mathrm{H}_{36} \mathrm{O}$ & 284.84 & White & $121-124$ & $0.37^{\mathrm{a}}$ & 71.0 \\
\hline Int-iii & $\mathrm{C}_{17} \mathrm{H}_{31} \mathrm{~N}_{2} \mathrm{OS}$ & 312.51 & Yellow & $154-157$ & $0.42^{\mathrm{a}}$ & 52.1 \\
\hline Int-iv & $\mathrm{C}_{17} \mathrm{H}_{34} \mathrm{~N}_{4} \mathrm{~S}$ & 326.54 & Pinkish white & 178-181 & $0.41^{b}$ & 46.0 \\
\hline $\mathrm{T}_{1}$ & $\mathrm{C}_{24} \mathrm{H}_{38} \mathrm{~N}_{4} \mathrm{~S}$ & 414.65 & Creamish white & 184-187 & $0.40^{\mathrm{b}}$ & 78.0 \\
\hline $\mathrm{T}_{2}$ & $\mathrm{C}_{25} \mathrm{H}_{38} \mathrm{~N}_{4} \mathrm{OS}$ & 442.66 & Light brown & $182-185$ & $0.33^{b}$ & 82.0 \\
\hline $\mathrm{T}_{3}$ & $\mathrm{C}_{25} \mathrm{H}_{40} \mathrm{~N}_{4} \mathrm{~S}$ & 428.68 & Yellowish white & 190-193 & $0.52^{\mathrm{b}}$ & 74.0 \\
\hline $\mathrm{T}_{4}$ & $\mathrm{C}_{26} \mathrm{H}_{43} \mathrm{~N}_{4} \mathrm{O}_{2} \mathrm{~S}$ & 474.70 & White & 188-191 & $0.60^{b}$ & 85.0 \\
\hline $\mathrm{T}_{5}$ & $\mathrm{C}_{27} \mathrm{H}_{44} \mathrm{~N}_{4} \mathrm{O}_{3} \mathrm{~S}$ & 504.73 & Yellow & $187-190$ & $0.38^{\mathrm{b}}$ & 79.5 \\
\hline $\mathrm{T}_{6}$ & $\mathrm{C}_{25} \mathrm{H}_{40} \mathrm{~N}_{4} \mathrm{O}_{2} \mathrm{~S}$ & 460.68 & Creamish white & 189-192 & $0.49^{b}$ & 84.6 \\
\hline $\mathrm{T}_{7}$ & $\mathrm{C}_{24} \mathrm{H}_{38} \mathrm{~N}_{4} \mathrm{OS}$ & 430.65 & Lemon yellow & $181-184$ & $0.32^{\mathrm{b}}$ & 78.0 \\
\hline $\mathrm{T}_{8}$ & $\mathrm{C}_{24} \mathrm{H}_{38} \mathrm{~N}_{4} \mathrm{OS}$ & 430.65 & Greenish white & 192-195 & $0.73^{b}$ & 65.9 \\
\hline $\mathrm{T}_{9}$ & $\mathrm{C}_{26} \mathrm{H}_{43} \mathrm{~N}_{5} \mathrm{~S}$ & 457.72 & Green & 198-201 & $0.62^{b}$ & 75.0 \\
\hline $\mathrm{T}_{10}$ & $\mathrm{C}_{24} \mathrm{H}_{37} \mathrm{ClN}_{4} \mathrm{~S}$ & 449.10 & Pinkish white & $205-208$ & $0.59^{b}$ & 79.6 \\
\hline $\mathrm{T}_{11}$ & $\mathrm{C}_{24} \mathrm{H}_{37} \mathrm{ClN}_{4} \mathrm{~S}$ & 449.10 & White & $202-205$ & $0.65^{b}$ & 78.0 \\
\hline $\mathrm{T}_{12}$ & $\mathrm{C}_{24} \mathrm{H}_{36} \mathrm{Cl}_{2} \mathrm{~N}_{4} \mathrm{~S}$ & 483.54 & White & $211-214$ & $0.34^{b}$ & 74.3 \\
\hline $\mathrm{T}_{13}$ & $\mathrm{C}_{24} \mathrm{H}_{37} \mathrm{BrN}_{4} \mathrm{~S}$ & 493.55 & White & $197-200$ & $0.39^{b}$ & 89.2 \\
\hline $\mathrm{T}_{14}$ & $\mathrm{C}_{24} \mathrm{H}_{37} \mathrm{BrN}_{4} \mathrm{~S}$ & 493.55 & Brownish yellow & 193-196 & $0.71^{b}$ & 85.8 \\
\hline $\mathrm{T}_{15}$ & $\mathrm{C}_{24} \mathrm{H}_{37} \mathrm{~N}_{5} \mathrm{O}_{2} \mathrm{~S}$ & 459.65 & Mustard yellow & 195-198 & $0.68^{\mathrm{b}}$ & 78.9 \\
\hline $\mathrm{T}_{16}$ & $\mathrm{C}_{24} \mathrm{H}_{37} \mathrm{~N}_{5} \mathrm{O}_{2} \mathrm{~S}$ & 459.65 & Dark yellow & 199-202 & $0.45^{b}$ & 90.0 \\
\hline $\mathrm{T}_{17}$ & $\mathrm{C}_{24} \mathrm{H}_{37} \mathrm{~N}_{5} \mathrm{O}_{2} \mathrm{~S}$ & 459.65 & Brown & $206-209$ & $0.50^{\mathrm{b}}$ & 85.8 \\
\hline $\mathrm{T}_{18}$ & $\mathrm{C}_{28} \mathrm{H}_{40} \mathrm{~N}_{4} \mathrm{OS}$ & 480.71 & Red & $186-189$ & $0.67^{b}$ & 88.8 \\
\hline $\mathrm{T}_{19}$ & $\mathrm{C}_{26} \mathrm{H}_{40} \mathrm{~N}_{4} \mathrm{OS}$ & 440.69 & Creamish white & 179-182 & $0.24^{b}$ & 87.9 \\
\hline$T_{20}$ & $\mathrm{C}_{22} \mathrm{H}_{36} \mathrm{~N}_{4} \mathrm{OS}$ & 404.61 & White & 196-199 & $0.43^{b}$ & 87.0 \\
\hline
\end{tabular}

Mobile phase: a[chloroform:toluene, $7: 3{ }^{\text {b }}{ }^{\mathrm{b}}$ [ethylacetate: $n$-hexane, 2:3]

\section{Experimental}

The initial material, reagents and solvents were purchased from Loba chemie. The glasswares were obtained from Borosil. The raw material was weighed on calibrated weighing balance. The synthetic scheme was drawn via ChemDraw 8.03. The confirmation of reaction at every step was done by TLC (thin layer chromatography). Melting point of the synthesized compounds was depicted by labtech melting point equipment. For spectral characterizations of the compounds, Bruker 12060280, Software: OPUS 7.2.139.1294 spectrometer using ATR for IR spectra $\left(\mathrm{cm}^{-1}\right)$ and Bruker Avance III at $600 \mathrm{NMR}$ and $150 \mathrm{MHz}$ for ${ }^{1} \mathrm{H}$ and ${ }^{13} \mathrm{CNMR}$ (DMSO-d6, $\delta \mathrm{ppm}$ ) were used. The tested microbial strains like Gram positive, Gram negative bacteria and fungi were obtained from the Institute of Microbial Technology and Gene bank, Chandigarh for the in vitro antimicrobial activity. Waters Micromass Q-ToF Micro instrument was used for mass spectra. Elemental analysis was performed on PerkinElmer $2400 \mathrm{C}, \mathrm{H}$ and $\mathrm{N}$ analyzer and all synthesized compounds gave $\mathrm{C}, \mathrm{H}$ and $\mathrm{N}$ analysis within $\pm 0.4 \%$ of the theoretical results.
Procedure for synthesized 1,2,4-triazole derivatives $\left(T_{1}-\right.$

$\mathrm{T}_{20}$ )

Step A: synthesis of Int-i

A mixture of palmitic acid (2.6 g, $0.01 \mathrm{~mol})$, absolute ethanol $(50 \mathrm{ml})$ and few drops of conc. sulphuric acid $(0.5 \mathrm{ml})$ was refluxed for $10 \mathrm{~h}$ in a round bottom flask and then cooled to $5{ }^{\circ} \mathrm{C}$. The liquid product was separated from reaction mixture by using ether on the basis of density and then purified [26].

\section{Step B: synthesis of Int-ii}

To a solution of ethyl palmitate (Int-i, $2.8 \mathrm{~g}, 0.01 \mathrm{~mol}$ ) in absolute ethanol $(30 \mathrm{ml})$, hydrazine hydrate $(0.64 \mathrm{~g}$, $0.02 \mathrm{~mol}$ ) was added and refluxed for $6 \mathrm{~h}$ and then left to cool. The solid product was collected by filtration and recrystallized from ethanol [26].

\section{Step C: synthesis of Int-iii}

Palmitohydrazide (Int-ii, $3.12 \mathrm{~g}, 0.01 \mathrm{~mol}$ ) dissolved in the solution of potassium hydroxide $(1.12 \mathrm{~g}, 0.02 \mathrm{~mol})$ in ethanol $(30 \mathrm{ml})$ and then $(0.76 \mathrm{~g}, 0.01 \mathrm{~mol})$ carbon disulfide was added slowly in the reaction mixture. The reaction mixture was refluxed for 10-12 hand then cooled at room temperature followed by addition of 
Table 2 Spectral characterization of synthesized derivatives $\left(T_{1}-T_{20}\right)$

\section{Comp IR $\left(\mathrm{KBr}, \mathrm{cm}^{-1}\right)$ \\ ${ }^{1}$ H NMR (400 MHz, DMSO- $d_{6}$ ) \\ ${ }^{13} \mathrm{C}$ NMR (400 MHz, DMSO- $\left.d_{6}\right)$}

$1.22-2.24\left(\mathrm{~m}, 28 \mathrm{H}, \mathrm{CH}_{2}\right), 0.83(\mathrm{~s}, 3 \mathrm{H}$, $\left.\mathrm{CH}_{3}\right), 2.5\left(\mathrm{~s}, 2 \mathrm{H}, \mathrm{NH}_{2}\right), 8.873(\mathrm{~s}, 1 \mathrm{H}, \mathrm{NH})$
$\mathrm{C}, \mathrm{H}, \mathrm{N}$ analyses calculated (found); MS, ES + (ToF): $m / z-\left[M^{+}+1\right]$

14.0, 19.4, 29.4, 39.1, 128.9, $149.9,168.6,189.4$

$1.22-2.47\left(\mathrm{~m}, 28 \mathrm{H}, \mathrm{CH}_{2}\right), 0.84\left(\mathrm{~s}, 3 \mathrm{H}, \mathrm{CH}_{3}\right) 15.7,18.7,29.8,37.1,124.1$, $149.9,160.6,182.4$

$17.7,18.4,29.8,39.6,128.1$ $124.98,149.9,168.6,188.4$

$22-2.12\left(\mathrm{~m}, 28 \mathrm{H}_{1} \mathrm{CH}_{2}\right), 0.82\left(\mathrm{~s}, 3 \mathrm{H}_{1}\right.$ $\left.\mathrm{CH}_{3}\right), 2.51\left(\mathrm{~s}, 2 \mathrm{H}, \mathrm{NH}_{2}\right), 8.87(\mathrm{~s}, 1 \mathrm{H}, \mathrm{NH})$ 1372 (C=S str.), 1589 (C=N str.), 1115 (C-O str.)

Int-iv 3319 (N-H str.), 2919 (C-H str., aliphatic), 1631 (C=N str.), 2572 (S-H str.)

2925 (C-H str., aliphatic), 1625 (C=N str.), $1.24-2.45\left(\mathrm{~m}, 28 \mathrm{H}, \mathrm{CH}_{2}\right), 0.84(\mathrm{~s}, 3 \mathrm{H}$, 3100 ( $\mathrm{C}=\mathrm{C}$ str., aromatic), $1719\left(\mathrm{C}-\mathrm{H}\right.$ str., $\left.\mathrm{CH}_{3}\right), 7.41-7.93(\mathrm{~m}, 5 \mathrm{H}, \mathrm{Ar}-\mathrm{H}), 3.37$ (s, aromatic.), 2568 (S-H str.) $\mathrm{H}, \mathrm{SH})$

$\mathbf{T}_{2}$ 2990 (C-H str., aliphatic), 1646 (C=N str.), 1751 ( $C=0$, str.), 1641 ( $C=C$ str., aromatic), 3073 (C-H str., aromatic), 2572 (S-H str.)

$1.32-2.51\left(\mathrm{~m}, 28 \mathrm{H}, \mathrm{CH}_{2}\right), 0.86(\mathrm{~s}, 3 \mathrm{H}$ $\left.\mathrm{CH}_{3}\right), 2.51\left(\mathrm{~s}, 2 \mathrm{H}, \mathrm{CH}_{2}\right), 7.08-7.91(\mathrm{~m}$, $4 \mathrm{H}, \mathrm{Ar}-\mathrm{H}), 3.37(\mathrm{~S}, \mathrm{H}, \mathrm{SH}), 10.02(\mathrm{~s}, \mathrm{H}$, $\mathrm{CHO})$

$\mathbf{T}_{3} 2922$ (C-H str., aliphatic), 1649 ( $\mathrm{C}=\mathrm{N}$ str.), $1.29-2.51\left(\mathrm{~m}, 24 \mathrm{H}, \mathrm{CH}_{2}\right), 0.87(\mathrm{~s}, 3 \mathrm{H}$, 1646 (C=C str., aromatic), $3098(\mathrm{C}-\mathrm{H}$ str., aromatic), 1790 (ring, str.), 2560 (S-H str.)

$\mathbf{T}_{4} 2920$ (C-H str., aliphatic), 1631 ( $\mathrm{C}=\mathrm{N}$ str.), 2856 (C-O-C str.), 1647 ( $C=$ C str., aromatic), 3105 (C-H str., aromatic), 2593 (S-H str)

$\mathbf{T}_{5} 2923$ (C-H str., aliphatic), 1639 (C=N str.), 2860 (C-O-C str.), 1450 ( $C=$ C str., aromatic), 3078 (C-H str., aromatic), 2572 (S-H str.) $\left.\mathrm{CH}_{3}\right), 7.30(\mathrm{~s}, \mathrm{H},=\mathrm{CH}), 7.51-7.62(\mathrm{~m}$ $4 \mathrm{H}, \mathrm{Ar}-\mathrm{H}), 3.37(\mathrm{~s}, \mathrm{H}, \mathrm{SH}), 2.34(\mathrm{~s}, 3 \mathrm{H}$, $\left.\mathrm{Ar}-\mathrm{CH}_{3}\right)$

$1.26-2.51\left(\mathrm{~m}, 28 \mathrm{H}, \mathrm{CH}_{2}\right), 0.86(\mathrm{~s}, 3 \mathrm{H}$, $\left.\mathrm{CH}_{3}\right), 7.90(\mathrm{~s}, \mathrm{H},=\mathrm{CH}), 7.01-7.61(\mathrm{~m}$, $3 \mathrm{H}, \mathrm{Ar}-\mathrm{H}), 3.11(\mathrm{~s}, \mathrm{H}, \mathrm{SH}), 3.76(\mathrm{~s}, 6 \mathrm{H}$, $\left.\mathrm{Ar}-\mathrm{OCH}_{3}\right)$

1.23-2.47 (m, 28H, $\left.\mathrm{CH}_{2}\right), 0.85(\mathrm{~s}, 3 \mathrm{H}$, $\left.\mathrm{CH}_{3}\right), 7.21(\mathrm{~s}, \mathrm{H},=\mathrm{CH}), 7.10-7.27(\mathrm{~m}$, $2 \mathrm{H}, \mathrm{Ar}-\mathrm{H}), 3.52(\mathrm{~s}, \mathrm{H}, \mathrm{SH}), 3.76(\mathrm{~s}, 9 \mathrm{H}$ $\left.\mathrm{Ar}-\mathrm{OCH}_{3}\right)$

$\mathrm{T}_{6}$

2988 (C-H str., aliphatic), 1677 (C=N str.), 1715 (ring, str.), 3400 (O-H str.), 2843 (C-O-C str.), 1636 (C=C str., aromatic), 3154 (C-H str., aromatic), 2555 (S-H str.)

$\mathbf{T}_{7} 2923$ (C-H str., aliphatic), 1617 (C=N str.), $3401(\mathrm{O}-\mathrm{H}, \mathrm{str}$ ), 1632 ( $\mathrm{C}=\mathrm{C}$ str., aromatic), 3101 (C-H str., aromatic), 2695 (S-H str.)

T8 2919 (C-H str., aliphatic), 1686 (C=N str.), 3483 (O-H, str.), 1665 (C=C str., aromatic), 3098 (C-H str., aromatic), 2572 (S-H str.)

$1.21-2.46\left(\mathrm{~m}, 28 \mathrm{H}, \mathrm{CH}_{2}\right), 0.96(\mathrm{~s}, 3 \mathrm{H}$, $\left.\mathrm{CH}_{3}\right), 8.0(\mathrm{~s}, \mathrm{H}, \mathrm{CH}), 7.51-7.69(\mathrm{~m}, 3 \mathrm{H}$, $\mathrm{Ar}-\mathrm{H}), 3.37(\mathrm{~s}, \mathrm{H}, \mathrm{SH}), 6.71(\mathrm{~s}, \mathrm{H}, \mathrm{OH})$, $3.79\left(\mathrm{~s}, \mathrm{H}, \mathrm{Ar}-\mathrm{OCH}_{3}\right)$

1.27-2.52 (m, 28H, CH $), 0.85(\mathrm{~s}, 3 \mathrm{H}$, $\left.\mathrm{CH}_{3}\right), 8.35(\mathrm{~s}, \mathrm{H},=\mathrm{CH}), 7.31-7.79 \mathrm{~m}, 4 \mathrm{H}$ $\mathrm{Ar}-\mathrm{H}), 3.37(\mathrm{~s}, \mathrm{H}, \mathrm{SH}), 4.05(\mathrm{~s}, \mathrm{H}, \mathrm{OH})$

$1.28-2.53\left(\mathrm{~m}, 28 \mathrm{H}, \mathrm{CH}_{2}\right), 0.88(\mathrm{~s}, 3 \mathrm{H}$, $\left.\mathrm{CH}_{3}\right), 8.10(\mathrm{~s}, \mathrm{H},=\mathrm{CH}), 6.43-7.49(\mathrm{~m}$, $4 \mathrm{H}, \mathrm{Ar}-\mathrm{H}), 3.37$ (s, H, SH), $5.01(\mathrm{~s}, \mathrm{H}$, $\mathrm{OH})$

T9 2925 (C-H str., aliphatic), 1684 (C=N str.), $1.21-2.51\left(\mathrm{~m}, 28 \mathrm{H}, \mathrm{CH}_{2}\right), 0.85(\mathrm{~s}, 3 \mathrm{H}$, 1645 (C=C str., aromatic), 3097 (C-H str., $\left.\mathrm{CH}_{3}\right), 7.11-7.48(\mathrm{~m}, 4 \mathrm{H}, \mathrm{Ar}-\mathrm{H}), 8.12$ (s, aromatic), $3431(\mathrm{~N}-\mathrm{H}$ amine, str.) $2572 \mathrm{H},=\mathrm{CH}), 3.01(\mathrm{~s}, \mathrm{H}, \mathrm{SH}), 2.99(\mathrm{~s}, 6 \mathrm{H}$, (S-H str.) $\left.\mathrm{N}-\left(\mathrm{CH}_{3}\right)_{2}\right)$

$\mathbf{T}_{10} 2916$ (C-H str., aliphatic), 1650 (C=N str.), $1.24-2.45\left(\mathrm{~m}, 28 \mathrm{H}, \mathrm{CH}_{2}\right), 0.85(\mathrm{~s}, 3 \mathrm{H}$ $1456\left(\mathrm{C}=\mathrm{C}\right.$ str., aromatic), $3065\left(\mathrm{C}-\mathrm{H}\right.$ str., $\left.\mathrm{CH}_{3}\right), 8.01(\mathrm{~s}, \mathrm{H},=\mathrm{CH}), 6.80-7.61(\mathrm{~m}$, aromatic), 750 (C-Cl str.), 2567 (S-H str.) 4H, Ar-H), 3.0 (s, H, SH)
$15.0,18.4,29.1,39.1,124.1$ $128.98,148.9,160.6,182.4$

$13.8,22.0,28.5-28.9,31.2$ $129.8,139.8,140.9,174.6$, 192.4

13.8, 22.0, 24.4, 28.5-28.8, 31.2,C, 70.05; H, 9.41; N, 13.07 (C, $128.6,129.7,142.3,161.11 \quad 70.00 ; H, 9.38 ; N, 13.02) ; 429$

13.8, 22.0, 28.3-28.9, 31.2, 55.1, C, 65.78; H, 8.92; N, 11.80; (C, $111.1,149.1,176.0$ 65.72; $\mathrm{H}, 8.89 ; \mathrm{N}, 11.78 ; \mathrm{O}$, $6.70) ; 475$

14.0, 39.1, 105.6, 129.2, 140.2, C, 64.25; H, 8.79; N, 11.10; (C, $153.1,161.1$ $64.21 ; H, 8.75 ; \mathrm{N}, 11.08) ; 505$

$13.8,22.0,28.5-28.9,31.8,55.5, C, 65.18 ; H, 8.75 ; N, 12.16$; (C, $115.3,120.7,128.7,142.7, \quad 65.13 ; H, 8.78 ; N, 12.15) ; 461$ $146.2,168.2$

14.0, 15.0, 24.4, 29.0, 31.2, 33.9, C, 66.94; H, 8.89; N, 13.01; (C, $39.9,59.9,61.1,116.6,129.4, \quad 66.90 ; H, 8.83 ; N, 12.9) ; 431$ $157.06,162.7$

14.3, 24.7, 29.0, 31.6, 34.1, 39.9, C, 66.94; H, 8.89; N, 13.01; (C, $46.2,116.2,129.1,160,175, \quad 66.90 ; \mathrm{H}, 8.85 ; \mathrm{N}, 12.89) ; 431$ 191.5

$13.8,22.0,24.4,29.0,31.2,46.3, \mathrm{C}, 68.23 ; \mathrm{H}, 9.47 ; \mathrm{N}, 15.30$ (C, $57.6,118.7,124.9,128.1, \quad 68.20 ; \mathrm{H}, 9.42 ; \mathrm{N}, 15.27) ; 458$ $130.1,134.9,168.1,114.4$

14.2, 22.2, 24.5, 29.4, 31.0, 46.3, C, 64.19; H, 8.30; N, 12.48; (C,

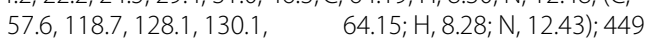
$134.9,148,168.1$

$\mathbf{T}_{11} 2916$ (C-H str., aliphatic), 1650 (C=N str.), $1.26-2.56\left(\mathrm{~s}, 28 \mathrm{H}, \mathrm{CH}_{2}\right), 0.87\left(\mathrm{~s}, 3 \mathrm{H}, \mathrm{CH}_{3}\right)$, 1635 ( $\mathrm{C}=\mathrm{C}$ str., aromatic), 3105 ( $\mathrm{C}-\mathrm{H}$ str., $8.01(\mathrm{~s}, \mathrm{H},=\mathrm{CH}), 6.90-7.68(\mathrm{~m}, 4 \mathrm{H}$, aromatic), 767 (C-Cl str.), 2572 (S-H str.) $\mathrm{Ar}-\mathrm{H}), 3.2(\mathrm{~s}, \mathrm{H}, \mathrm{SH})$

$\mathbf{T}_{12} 2905$ (C-H str., aliphatic), $1678.98\left(\mathrm{C}=\mathrm{N} \quad 1.23-2.51\left(\mathrm{~m}, 28 \mathrm{H}, \mathrm{CH}_{2}\right), 0.86(\mathrm{~s}, 3 \mathrm{H}\right.$, str.), 1604 ( $\mathrm{C}=\mathrm{C}$ str., aromatic), $\left.3087 \quad \mathrm{CH}_{3}\right), 8.10(\mathrm{~s}, \mathrm{H},=\mathrm{CH}), 6.60-7.57(\mathrm{~m}$ (C-H str., aromatic), 767 (C-Cl str.), $25053 \mathrm{H}, \mathrm{Ar}-\mathrm{H}), 3.07$ (s, H, SH) (S-H str.)

14.1, 22.0, 24.2, 29.9, 31.9, 40.3, C, 64.19; H, 8.30; N, 12.48; (C, $55.6,118.7,128.1,131.1, \quad 64.15 ; \mathrm{H}, 8.27 ; \mathrm{N}, 12.43) ; 449$ $137.9,148.9,168.8$

$13.9,22.5,24.4,29.9,31.5,44.3, C, 59.61 ; H, 7.50 ; \mathrm{N}, 11.59 ;(C$, $57.6,119.7,127.1,131.9, \quad 59.58 ; H, 7.48 ; N, 11.53) ; 483$ $139.9,148.9,168.9$

14.4, 23.2, 24.0, 29.9, 33.3, 48.3, C, 58.41; H, 7.56; N, 11.35; (C, $59.6,118.7,128.9,130.9, \quad 58.41 ; H, 7.56 ; N, 11.35) ; 495$ $134.8,148.7,168.6$ 
Table 2 (continued)

Comp IR $\left(\mathrm{KBr}, \mathrm{cm}^{-1}\right)$
${ }^{1} \mathrm{H}$ NMR (400 MHz, DMSO- $d_{6}$ )

\section{${ }^{13} \mathrm{C}$ NMR (400 MHz, DMSO- $d_{6}$ )}

$\mathrm{C}, \mathrm{H}, \mathrm{N}$ analyses calculated (found); MS, ES + (ToF): $m / z-\left[\mathrm{M}^{+}+1\right]$
$\mathbf{T}_{14} 2919$ (C-H str., aliphatic), 1648 ( $\mathrm{C}=\mathrm{N}$ str.), $1.29-2.59\left(\mathrm{~m}, 28 \mathrm{H}, \mathrm{CH}_{2}\right), 0.88$ (s, 3H, 1498 ( $\mathrm{C}=\mathrm{C}$ str., aromatic), $3054\left(\mathrm{C}-\mathrm{H}\right.$ str., $\left.\mathrm{CH}_{3}\right), 8.98(\mathrm{~s}, \mathrm{H},=\mathrm{CH}), 6.50-7.68(\mathrm{~m}$, aromatic), 699 (C-Br str.), 2505 (S-H str) 4H, Ar-H), 3.09 (s, H, SH)

$\mathbf{T}_{15} 2921$ (C-H str., aliphatic), 1698 ( $\mathrm{C}=\mathrm{N}$ str.), $1.29-2.67\left(\mathrm{~m}, 28 \mathrm{H}, \mathrm{CH}_{2}\right), 0.87(\mathrm{~s}, 3 \mathrm{H}$ $1445\left(\mathrm{C}=\mathrm{C}\right.$ str., aromatic), $3100\left(\mathrm{C}-\mathrm{H}\right.$ str., $\left.\mathrm{CH}_{3}\right), 8.02(\mathrm{~s}, \mathrm{H},=\mathrm{CH}), 7.51-7.64(\mathrm{~m}$, aromatic), 1545 ( $\mathrm{NO}_{2}$ str.), 2529 (S-H str.) $\left.4 \mathrm{H}, \mathrm{Ar}-\mathrm{H}\right), 3.07$ (s, H, SH),

$\mathbf{T}_{16} 2878$ ( $\mathrm{C}-\mathrm{H}$ str., aliphatic), 1648 ( $\mathrm{C}=\mathrm{N}$ str.) $), 1.29-2.51\left(\mathrm{~m}, 28 \mathrm{H}, \mathrm{CH}_{2}\right), 0.87(\mathrm{~s}, 3 \mathrm{H}$ $1646\left(\mathrm{C}=\mathrm{C}\right.$ str., aromatic), $3045\left(\mathrm{C}-\mathrm{H}\right.$ str., $\left.\mathrm{CH}_{3}\right), 8.01(\mathrm{~s}, \mathrm{H}, \mathrm{CH}), 7.51-7.96(\mathrm{~m}$, aromatic), 1424 ( $\mathrm{NO}_{2}$ str.), 2572 (S-H str.) $\left.4 \mathrm{H}, \mathrm{Ar}-\mathrm{H}\right), 3.37$ (s, H, SH), 2.34 (s, 3H,

$$
\left.\mathrm{Ar}-\mathrm{CH}_{3}\right)
$$

$\mathbf{T}_{17} 2880$ (C-H str., aliphatic), 1647 ( $\mathrm{C}=\mathrm{N}$ str.), $1.24-2.56\left(\mathrm{~m}, 28 \mathrm{H}, \mathrm{CH}_{2}\right), 0.86(\mathrm{~s}, 3 \mathrm{H}$, $1498\left(\mathrm{C}=\mathrm{C}\right.$ str., aromatic), $3076\left(\mathrm{C}-\mathrm{H}\right.$ str., $\left.\mathrm{CH}_{3}\right), 8.31(\mathrm{~s}, \mathrm{H},=\mathrm{CH}), 6.90-7.76(\mathrm{~m}$ aromatic), 1520 ( $\mathrm{NO}_{2}$ str.), $2572(\mathrm{~S}-\mathrm{H}$ str.) $\quad 4 \mathrm{H}, \mathrm{Ar}-\mathrm{H}), 3.07$ (s, H, SH)

$\mathbf{T}_{18} 2880$ (C-H str., aliphatic), 1650 ( $\mathrm{C}=\mathrm{N}$ str.) $), 1.29-2.98\left(\mathrm{~m}, 28 \mathrm{H}, \mathrm{CH}_{2}\right), 0.87(\mathrm{~s}, 3 \mathrm{H}$ $1608\left(\mathrm{C}=\mathrm{C}\right.$ str., aromatic), $3109\left(\mathrm{C}-\mathrm{H} \quad \mathrm{CH}_{3}\right), 8.01(\mathrm{~s}, \mathrm{H},=\mathrm{CH}), 6.88-8.08(\mathrm{~m}$ str., aromatic), 3401 (O-H str.), 2572 (S-H str.) $6 \mathrm{H}, \mathrm{Ar}-\mathrm{H}), 3.07(\mathrm{~s}, \mathrm{H}, \mathrm{SH}), 5.01(\mathrm{~s}, \mathrm{H}$, $\mathrm{Ar}-\mathrm{OH})$
$15.4,23.8,24.9,29.9,33.0,47.3, C, 58.41 ; H, 7.56 ; \mathrm{N}, 11.35 ;(\mathrm{C}$ $56.6,116.7,129.9,131.2, \quad 58.39 ; H, 7.53 ; \mathrm{N}, 11.30) ; 495$ $134.8,148.8,168.9$

$13.4,23.7,24.5,29.9,32.3,48.6, C, 62.71 ; H, 8.11 ; \mathrm{N}, 15.24$; (C, $59.8,118.9,127.9,131.9, \quad 62.69 ; \mathrm{H}, 8.09 ; \mathrm{N}, 15.20) ; 460$ $134.9,148.7,168.5$

$13.4,23.7,24.5,29.9,32.3,48.6, C, 62.71 ; H, 8.11 ; N, 15.24$; (C, $59.8,118.9,127.9,131.9, \quad 62.68 ; \mathrm{H}, 8.07 ; \mathrm{N}, 15.19) ; 460$ $134.9,148.7$

$14.4,23.9,24.7,29.8,31.3,48.6, C, 62.71 ; H, 8.11 ; \mathrm{N}, 15.24$; (C, $57.8,116.9,127.9,131.7, \quad 62.68 ; \mathrm{H}, 8.10 ; \mathrm{N}, 15.22) ; 460$ $135.9,148.9,168.3$

$13.4,22.9,24.8,29.8,31.3,46.6, C, 69.96 ; \mathrm{H}, 8.39 ; \mathrm{N}, 11.66$; (C, $54.8,116.9,126.9,130.7, \quad 69.91 ; H, 8.32 ; \mathrm{N}, 11.64) ; 481$ $135.9,148.9,169.3,189.9$

C, 70.86; H, 9.15; N, 12.71; (C, $70.82 ; \mathrm{H}, 9.12 ; \mathrm{N}, 12.62) ; 441$ 1 $22.1,29.5,31.2,54.8$ $116.9,126.9,130.7,135.9$ $148.6,164.3,189.6$

14.7, 21.9, 25.8, 26.8, 31.1, 47.6, C, 65.31; H, 8.97; N, 13.85; (C,

$54.5,119.9,130.1,135.5, \quad 65.28 ; \mathrm{H}, 8.91 ; \mathrm{N}, 13.80) ; 405$ $158.9,179.3,188.9$ str.), 2879 (C-O-C, str.), 1678 (C=C str. aromatic), 3156 (C-H str., aromatic), 2572 (S-H str) $1.29-2.52\left(\mathrm{~m}, 28 \mathrm{H}, \mathrm{CH}_{2}\right), 0.84(\mathrm{~s}, 3 \mathrm{H}$ $\left.\mathrm{CH}_{3}\right), 6.30-7.40(\mathrm{~m}, 3 \mathrm{H}, \mathrm{Ar}-\mathrm{H}), 3.07(\mathrm{~s}$ $\mathrm{H}, \mathrm{SH}$, 
Table 3 Antimicrobial screening results of the synthesized 1,2,4-triazole derivatives $\left(T_{1}-T_{20}\right)$

\begin{tabular}{|c|c|c|c|c|c|}
\hline \multirow[t]{4}{*}{ Compound } & \multicolumn{5}{|c|}{ Minimum inhibitory concentration $(\mu \mathrm{M})$} \\
\hline & \multicolumn{3}{|c|}{ Bacterial strain } & \multirow{2}{*}{\multicolumn{2}{|c|}{ Fungal strain }} \\
\hline & \multirow{2}{*}{$\begin{array}{l}\text { Gram +ve } \\
\text { B. substilis }\end{array}$} & \multicolumn{2}{|l|}{ Gram - ve } & & \\
\hline & & P. aeruginosa & E. coli & C. albican & A. niger \\
\hline $\mathrm{T}_{1}$ & 120.5 & 241.1 & 241.1 & 241.1 & 120.5 \\
\hline $\mathbf{T}_{2}$ & 56.4 & 56.4 & 112.9 & 225.9 & 225.9 \\
\hline $\mathrm{T}_{3}$ & 58.3 & 58.3 & 116.6 & 233.2 & 116.6 \\
\hline $\mathrm{T}_{4}$ & 52.6 & 52.6 & 105.3 & 52.6 & 105.3 \\
\hline $\mathrm{T}_{5}$ & 24.7 & 12.3 & 24.7 & 12.3 & 99.0 \\
\hline $\mathrm{T}_{6}$ & 108.5 & 54.2 & 54.2 & 108.5 & 217.0 \\
\hline $\mathrm{T}_{7}$ & 116.1 & 29.0 & 116.1 & 232.2 & 232.2 \\
\hline $\mathrm{T}_{8}$ & 116.1 & 58.0 & 58.0 & 116.1 & 232.2 \\
\hline $\mathrm{T}_{9}$ & 54.6 & 27.3 & 54.6 & 109.2 & 218.4 \\
\hline $\mathrm{T}_{10}$ & 55.6 & 55.6 & 111.3 & 222.6 & 55.6 \\
\hline$T_{11}$ & 55.6 & 111.3 & 111.3 & 27.8 & 111.3 \\
\hline$T_{12}$ & 103.4 & 51.7 & 103.4 & 206.8 & 103.4 \\
\hline$T_{13}$ & 101.3 & 202.6 & 202.6 & 101.3 & 202.6 \\
\hline $\mathrm{T}_{14}$ & 50.6 & 101.3 & 101.3 & 202.6 & 101.3 \\
\hline $\mathrm{T}_{15}$ & 54.3 & 108.7 & 217.5 & 217.5 & 54.3 \\
\hline $\mathrm{T}_{16}$ & 108.7 & 108.7 & 217.5 & 217.5 & 217.5 \\
\hline$T_{17}$ & 54.3 & 217.5 & 217.5 & 108.7 & 27.1 \\
\hline $\mathrm{T}_{18}$ & 104.0 & 52.0 & 104.0 & 208.0 & 104.0 \\
\hline $\mathrm{T}_{19}$ & 113.4 & 226.9 & 56.7 & 113.4 & 113.4 \\
\hline $\mathrm{T}_{20}$ & 61.7 & 123.5 & 61.7 & 247.1 & 247.1 \\
\hline Fluconazole & - & - & - & 40.8 & 20.4 \\
\hline Ciprofloxacin & 18.0 & 18.0 & 37.7 & - & - \\
\hline Amoxicillin & 17.1 & 17.1 & 17.1 & - & - \\
\hline
\end{tabular}

Italics signifies the most active compound in comparison to the standard compound

hydrochloric acid for neutralization of product. The precipitated solid was filtered, washed with ethanol, dried and recrystallized from ethanol [27].

\section{Step D: synthesis of Int-iv}

An ethanolic $(30 \mathrm{ml})$ solution of 5-pentadecyl-1,3,4oxadiazole-2(3H)-thione (Int-iii, $3.26 \mathrm{~g}, 0.01 \mathrm{~mol}$ ) and hydrazine hydrate $(0.38 \mathrm{~g}, 0.01 \mathrm{~mol})$ was heated under reflux for $3 \mathrm{~h}$ and then solution was poured in ice. The resulting product was filtered, washed and recrystallized from ethanol $[26,27]$.

\section{Step E: synthesis of 1,2,4-triazole derivatives $\left(T_{1}-T_{20}\right)$}

The reaction mixture of 4-amino-5-pentadecyl-4H-1,2,4triazole-3-thiol (Int-iv, $3.26 \mathrm{~g}, 0.01 \mathrm{~mol}$ ) and different substituted aldehydes $(0.01 \mathrm{~mol})$ in ethanol followed by addition of few drops of sulphuric acid was refluxed for an appropriate time. The reaction was monitored by thin layer chromatography. After completion of reaction, the product was poured in ice and filtered, then wash and finally solid products were collected and recrystallized from ethanol [27].

\section{Biological studies}

\section{Antimicrobial evaluation}

The in vitro antimicrobial screening of the synthesized 1,2,4-triazole derivatives $\left(\mathbf{T}_{\mathbf{1}}-\mathbf{T}_{\mathbf{2 0}}\right)$ in $\mu \mathrm{M}$ was determined against Gram-positive Bacillus subtilis, Pseudomonas aeruginosa, Gram-negative Escherichia coli bacterium and fungal strains Candida albicans and Aspergillus niger by tube dilution method using ciprofloxacin, amoxycillin (antibacterial) and fluconazole (antifungal) as reference drugs. DMSO was used to dissolve the reference and sample derivatives $\left(\mathbf{T}_{\mathbf{1}}-\mathbf{T}_{\mathbf{2 0}}\right)$. Dilutions were prepared in nutrient broth (I.P.) for bacterial (incubated at $37 \pm 1{ }^{\circ} \mathrm{C}$ for $24 \mathrm{~h}$ ) and Sabouraud dextrose broth (I.P.) for fungal species $\left(37 \pm 1{ }^{\circ} \mathrm{C}\right.$ for $48 \mathrm{~h}$ for $C$. albicans $)$ and $\left(25 \pm 1{ }^{\circ} \mathrm{C}\right.$ for 7 days for A. niger) (Table 3, Figs. 4 and 5) [17].

\section{In vitro antioxidant evaluation}

In the DPPH free radical scavenging activity, compounds $\left(\mathbf{T}_{1}-\mathbf{T}_{20}\right)$ were evaluated for their free radical scavenging activity with ascorbic acid as standard compound. The $\mathrm{IC}_{50}$ was calculated for each compound as well as ascorbic acid as standard and summarized in 

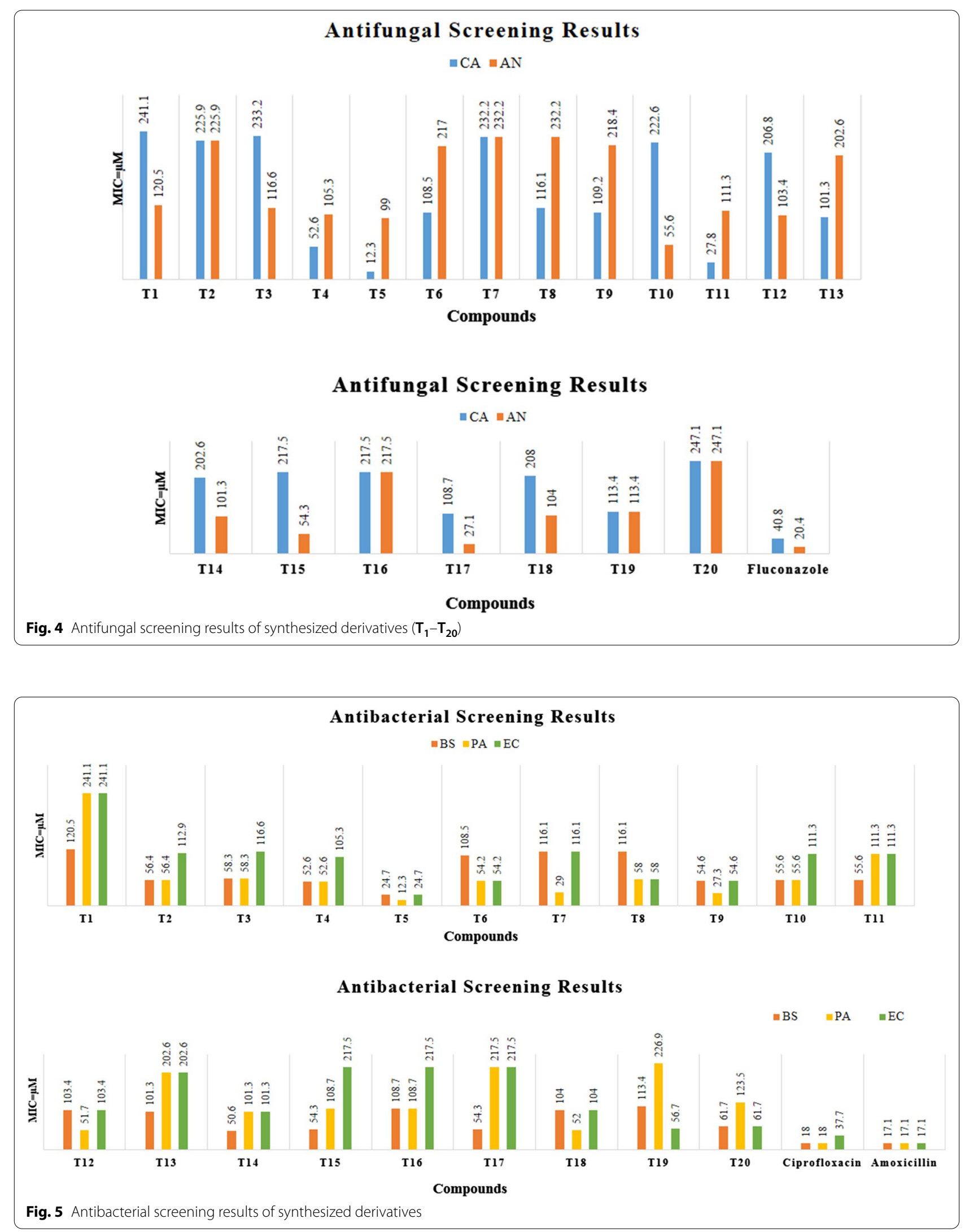
Table 4 and shown in Figs. 6, 7, 8. The scavenging effect increased with the increasing concentrations of sample compounds. DPPH is relatively stable nitrogen centered free radical that easily accepts an electron or hydrogen radical to become a stable diamagnetic molecule. DPPH radicals react with suitable reducing agents as a result of which the electrons become paired off forming the corresponding hydrazine. The solution therefore loses colour stoichometrically depending on the number of electrons taken up. Fifty millilitres of various concentrations (25, 50,70 and $100 \mu \mathrm{g} / \mathrm{ml}$ ) of the compounds dissolved in methanol was added to $5 \mathrm{ml}$ of a $0.004 \%$ methanolic solution of DPPH. The sample solutions were incubated for $30 \mathrm{~min}$ at room temperature in dark place and after then absorbance was recorded against the blank solution at $517 \mathrm{~nm}$. The relative percent of DPPH scavenging activity was calculated according to the following equation:

$$
\mathrm{I} \%=\frac{\mathrm{A}_{\text {control }}-\mathrm{A}_{\text {sample }}}{\mathrm{A}_{\text {control }}} \times 100,
$$

where $A_{\text {control }}$ is the absorbance of the control, $A_{\text {sample }}$ is the absorbance of the test compound.

\section{Urease inhibition evaluation}

Urease inhibitory potential for each synthesisized compound $\left(\mathbf{T}_{1}-\mathbf{T}_{20}\right)$ was evaluated using Jack Bean Urease by Indophenol method (Table 5, Figs. 9, 10, 11). $250 \mu \mathrm{l}$ of jack bean urease $(4 \mathrm{U})$ was mixed with $250 \mu \mathrm{l}$ of different synthesized test compounds and standard of different concentrations [dissolved in $\mathrm{DMSO} / \mathrm{H}_{2} \mathrm{O}$ mixture $(1: 1 \mathrm{v} / \mathrm{v})]$. The mixture was pre-incubated for $1 \mathrm{~h}$ at $37^{\circ} \mathrm{C}$ in test tubes. $2 \mathrm{ml}$ of $100 \mathrm{mM}$ phosphate buffer ( $\mathrm{pH}$ 6.8) containing $500 \mathrm{mM}$ urea and $0.002 \%$ phenol red as an indicator were added in sample test tubes after pre incubation and again incubated at room temperature. Absorbance of reaction mixture was recorded by ELISA at $570 \mathrm{~nm}$. Ammonium carbonate increased the $\mathrm{pH}$ of phosphate buffer from 6.8 to 7.7 which was produced from urea by urease enzyme and the end peak was measured by the colour of phenol red indicator [16].

The percentage inhibition of urease enzyme was calculated by using following formula:

$$
\mathrm{I} \%=\frac{\mathrm{A}_{\text {control }}-\mathrm{A}_{\text {sample }}}{\mathrm{A}_{\text {control }}} \times 100,
$$

\begin{tabular}{|c|c|c|c|c|c|}
\hline \multirow[t]{2}{*}{ Compounds } & \multicolumn{4}{|c|}{$\%$ inhibition } & \multirow[t]{2}{*}{$\mathrm{IC}_{50}(\mu \mathrm{g} / \mathrm{ml})$} \\
\hline & $25(\mu \mathrm{g} / \mathrm{ml})$ & $50(\mu \mathrm{g} / \mathrm{ml})$ & $75(\mu \mathrm{g} / \mathrm{ml})$ & $100(\mu \mathrm{g} / \mathrm{ml})$ & \\
\hline $\mathrm{T}_{1}$ & 25.45 & 49.65 & 69.67 & 94.78 & 46.83 \\
\hline $\mathrm{T}_{2}$ & 45.67 & 56.98 & 70.16 & 84.21 & 34.83 \\
\hline $\mathrm{T}_{3}$ & 43.56 & 60.12 & 75.67 & 88.98 & 34.38 \\
\hline $\mathrm{T}_{4}$ & 38.45 & 58.34 & 88.61 & 98.89 & 37.50 \\
\hline $\mathrm{T}_{5}$ & 42.34 & 55.67 & 68.98 & 78.12 & 39.13 \\
\hline $\mathrm{T}_{6}$ & 31.67 & 53.67 & 77.78 & 92.67 & 45.66 \\
\hline $\mathrm{T}_{7}$ & 46.75 & 52.56 & 60.56 & 70.41 & 38.54 \\
\hline $\mathrm{T}_{8}$ & 43.91 & 58.67 & 67.54 & 82.57 & 36.12 \\
\hline $\mathrm{T}_{9}$ & 40.56 & 60.67 & 83.61 & 92.16 & 35.42 \\
\hline$T_{10}$ & 45.56 & 51.56 & 57.57 & 62.67 & 43.57 \\
\hline$T_{11}$ & 35.78 & 52.89 & 70.89 & 89.9 & 45.36 \\
\hline$T_{12}$ & 44.45 & 51.45 & 68.56 & 70.61 & 39.56 \\
\hline$T_{13}$ & 43.46 & 57.67 & 73.76 & 89.56 & 36.40 \\
\hline$T_{14}$ & 37.56 & 60.68 & 85.79 & 95.61 & 37.52 \\
\hline $\mathrm{T}_{15}$ & 38.56 & 50.64 & 62.16 & 80.56 & 47.99 \\
\hline$T_{16}$ & 43.65 & 51.45 & 67.26 & 81.76 & 41.30 \\
\hline$T_{17}$ & 48.75 & 62.57 & 78.16 & 84.61 & 24.90 \\
\hline$T_{18}$ & 39.59 & 68.57 & 80.13 & 95.89 & 33.34 \\
\hline$T_{19}$ & 38.45 & 58.34 & 80.61 & 96.89 & 38.99 \\
\hline $\mathbf{T}_{20}$ & 33.45 & 53.67 & 70.46 & 92.67 & 46.34 \\
\hline Ascorbic acid & 38.67 & 63.68 & 84.78 & 94.45 & 35.44 \\
\hline
\end{tabular}

Table 4 Antioxidant screening results of the synthesized compounds $\left(T_{1}-T_{20}\right)$ 

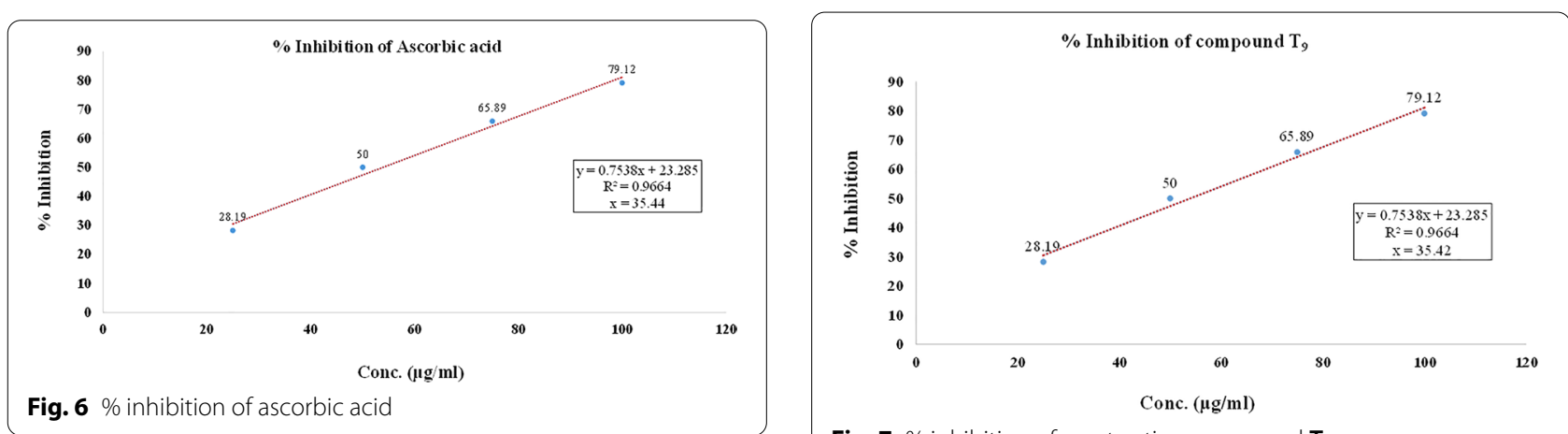

Fig. $7 \%$ inhibition of most active compound $\mathbf{T}_{\mathbf{9}}$

\section{Antioxidant Screening Results}

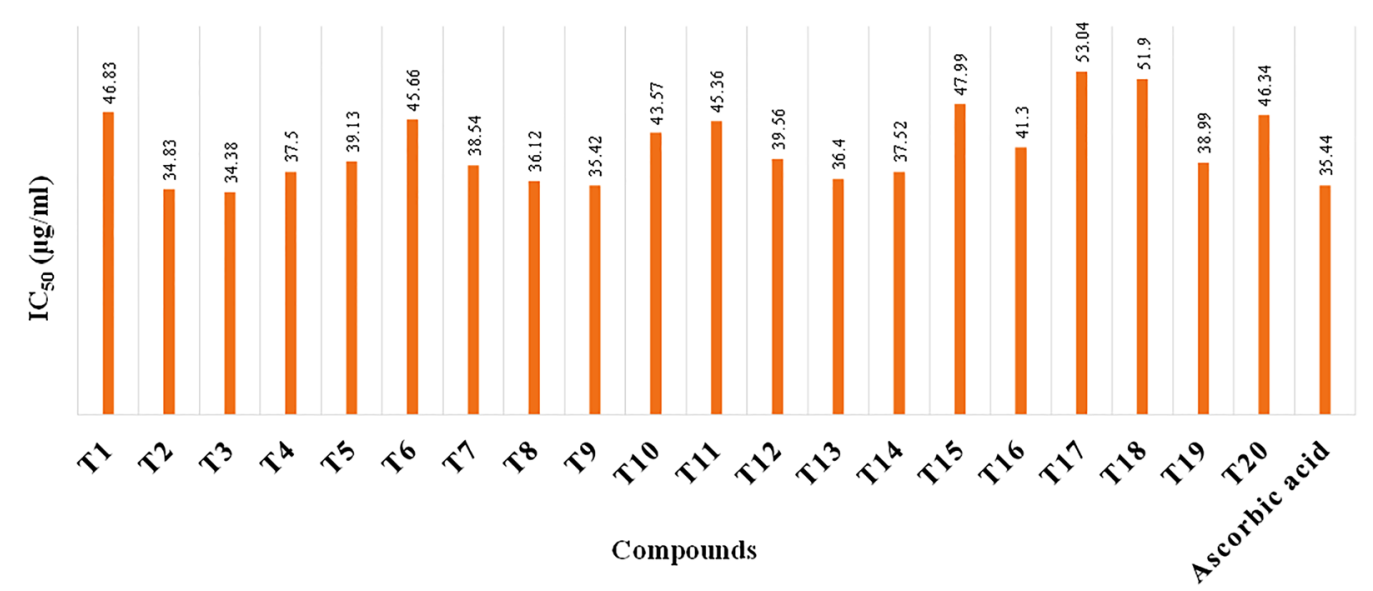

Fig. 8 Antioxidant screening results of synthesized derivatives $\left(\mathbf{T}_{\mathbf{1}}-\mathbf{T}_{\mathbf{2 0}}\right)$

where $\mathrm{A}_{\text {control }}$ is the absorbance of the control; $\mathrm{A}_{\text {sample }}$ is the absorbance of the test compound.

\section{Anticancer evaluation}

HCT116 (human colon cancer cells) were seeded at 2500 cells/well (96 well plate), allowed to attach overnight, exposed to the respective compounds for $72 \mathrm{~h}$ and subjected to SRB assay $(570 \mathrm{~nm})$. Data represent mean $\mathrm{IC}_{50}$ of at least triplicates. The compounds were all dissolved in DMSO as stock of $100 \mathrm{mg} / \mathrm{ml}$. DMSO of $<1.5 \%$ did not result in cell kill. The highest concentration of each compound tested $(100 \mu \mathrm{g} / \mathrm{ml})$ contained only $0.1 \%$ DMSO. Compounds $\mathbf{T}_{2}\left(\mathrm{IC}_{50}=3.84 \mu \mathrm{M}\right)$ and $\mathbf{T}_{7}\left(\mathrm{IC}_{50}=3.25 \mu \mathrm{M}\right)$ exhibited the most potent anticancer activity against HCT116 cell lines as compared to standard 5-FU $\left(\mathrm{IC}_{50}=25.36 \mu \mathrm{M}\right)$ given in Table 6 and Figs. 12, $13,14$. 
Table 5 Urease inhibitory screening results of the synthesized compounds $\left(T_{1}-T_{20}\right)$

\begin{tabular}{|c|c|c|c|c|c|}
\hline \multirow[t]{2}{*}{ Compounds } & \multicolumn{4}{|c|}{$\%$ inhibition } & \multirow[t]{2}{*}{$I C_{50}(\mu \mathrm{g} / \mathrm{ml})$} \\
\hline & $25(\mu \mathrm{g} / \mathrm{ml})$ & $50(\mu \mathrm{g} / \mathrm{ml})$ & $75(\mu \mathrm{g} / \mathrm{ml})$ & $100(\mu \mathrm{g} / \mathrm{ml})$ & \\
\hline $\mathrm{T}_{1}$ & 25.76 & 47.98 & 72.9 & 91.99 & 51.70 \\
\hline $\mathrm{T}_{2}$ & 40.45 & 49.54 & 55.67 & 67.57 & 53.04 \\
\hline $\mathrm{T}_{3}$ & 28.19 & 50.00 & 65.89 & 79.12 & 54.01 \\
\hline $\mathrm{T}_{4}$ & 35.45 & 49.45 & 63.67 & 79.45 & 50.52 \\
\hline $\mathrm{T}_{5}$ & 23.34 & 44.35 & 70.87 & 90.87 & 54.46 \\
\hline $\mathrm{T}_{6}$ & 38.45 & 50.34 & 65.61 & 80.89 & 47.02 \\
\hline $\mathrm{T}_{7}$ & 23.98 & 46.89 & 76.09 & 91.90 & 52.07 \\
\hline $\mathrm{T}_{8}$ & 22.45 & 39.58 & 68.58 & 92.67 & 56.43 \\
\hline $\mathrm{T}_{9}$ & 33.45 & 53.67 & 70.46 & 92.67 & 46.34 \\
\hline$T_{10}$ & 27.90 & 46.89 & 70.90 & 89.80 & 51.90 \\
\hline$T_{11}$ & 25.00 & 42.45 & 68.88 & 92.67 & 54.59 \\
\hline$T_{12}$ & 43.56 & 46.57 & 52.56 & 61.56 & 58.06 \\
\hline$T_{13}$ & 26.57 & 48.78 & 73.98 & 98.89 & 50.05 \\
\hline$T_{14}$ & 41.70 & 46.23 & 50.49 & 57.78 & 67.02 \\
\hline $\mathrm{T}_{15}$ & 29.03 & 37.14 & 61.78 & 73.12 & 61.04 \\
\hline$T_{16}$ & 45.76 & 49.10 & 53.87 & 59.12 & 52.85 \\
\hline$T_{17}$ & 37.91 & 42.38 & 57.61 & 71.42 & 57.47 \\
\hline$T_{18}$ & 40.90 & 46.79 & 55.89 & 67.9 & 54.30 \\
\hline$T_{19}$ & 31.89 & 47.98 & 52.98 & 65.87 & 63.24 \\
\hline $\mathrm{T}_{20}$ & 28.98 & 49.09 & 69.09 & 81.90 & 52.34 \\
\hline Thiourea & 29.98 & 46.76 & 67.78 & 76.78 & 54.25 \\
\hline
\end{tabular}

Italics signifies the most active compound in comparison to the standard compound
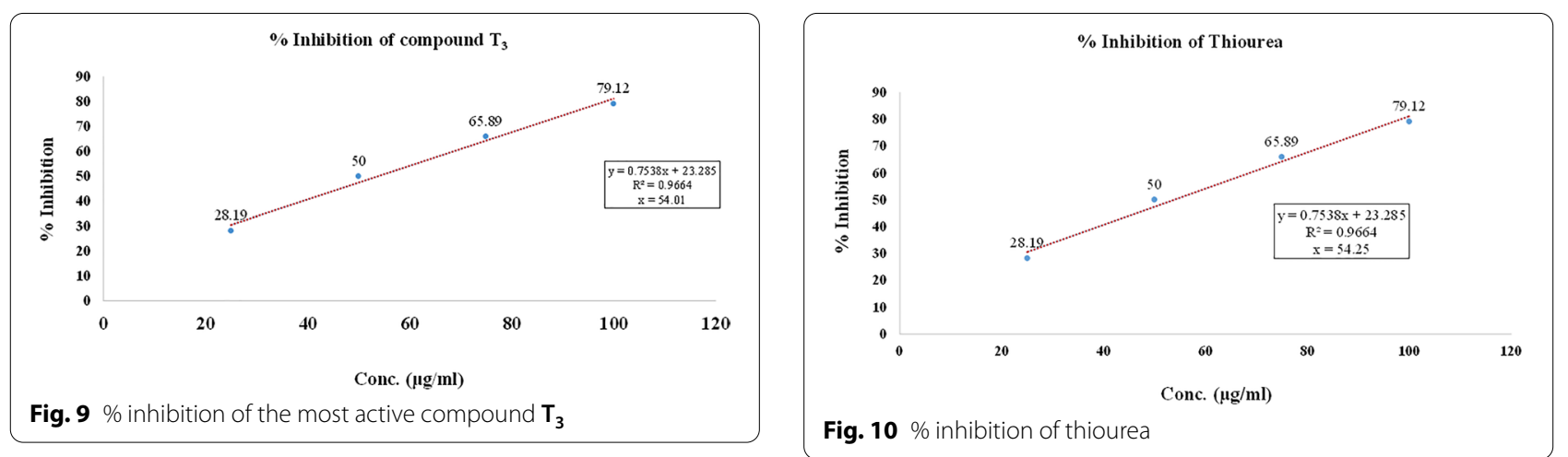


\section{Urease Inhibitory Screening Results}

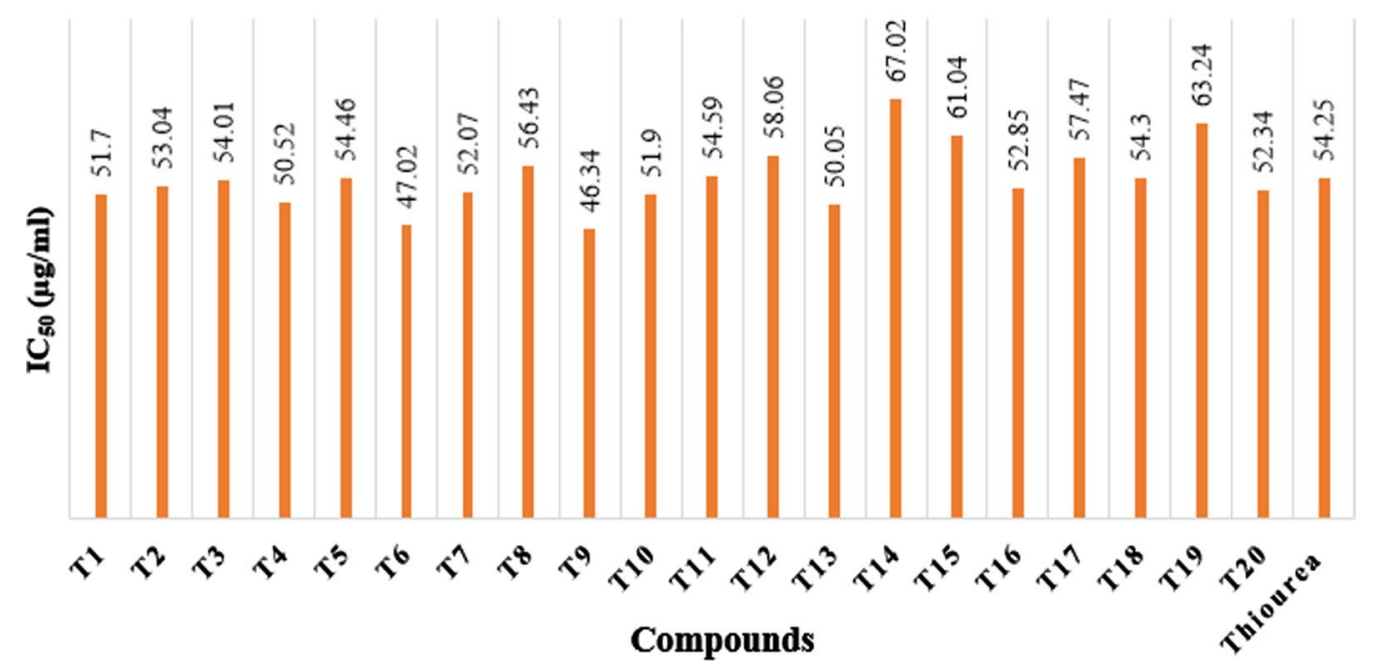

Fig. 11 Urease inhibitory screening results of the synthesized derivatives $\left(\mathbf{T}_{\mathbf{1}}-\mathbf{T}_{\mathbf{2 0}}\right)$

Table 6 Anticancer screening results of the synthesized compounds $\left(T_{1}-T_{20}\right)$

\begin{tabular}{|c|c|c|c|}
\hline Compounds & $I C_{50}(\mu \mathrm{M})$ & Compounds & $I C_{50}(\mu M)$ \\
\hline $\mathrm{T}_{1}$ & $>241.16$ & $\mathrm{~T}_{12}$ & $>206.80$ \\
\hline $\mathrm{T}_{2}$ & 3.84 & $\mathrm{~T}_{13}$ & $>202.61$ \\
\hline$T_{3}$ & 16.56 & $\mathrm{~T}_{14}$ & 128.25 \\
\hline $\mathrm{T}_{4}$ & 7.79 & $\mathrm{~T}_{15}$ & $>217.55$ \\
\hline $\mathrm{T}_{5}$ & $>198.12$ & $\mathrm{~T}_{16}$ & 169.25 \\
\hline$T_{6}$ & 112.00 & $T_{17}$ & $>217.55$ \\
\hline$T_{7}$ & 3.25 & $\mathrm{~T}_{18}$ & $>208.02$ \\
\hline $\mathrm{T}_{8}$ & $>232.20$ & $\mathrm{~T}_{19}$ & $>226.91$ \\
\hline$T_{9}$ & $>218.47$ & $\mathrm{~T}_{20}$ & $>247.15$ \\
\hline$T_{10}$ & 173.90 & 5-FU & 25.36 \\
\hline $\mathrm{T}_{11}$ & $>222.66$ & DMSO & $1.50 \%$ \\
\hline
\end{tabular}

Italics signifies the most active compound in comparison to the standard compound
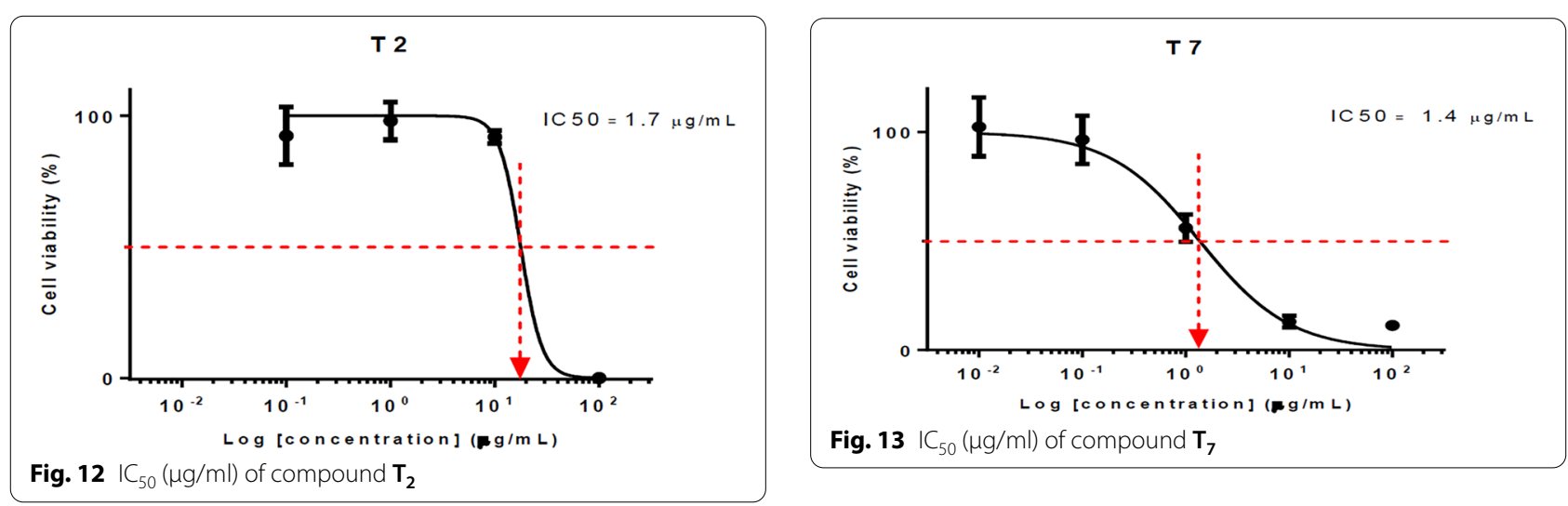

Fig. $13 \mathrm{IC}_{50}(\mu \mathrm{g} / \mathrm{ml})$ of compound $\mathbf{T}_{\mathbf{7}}$ 


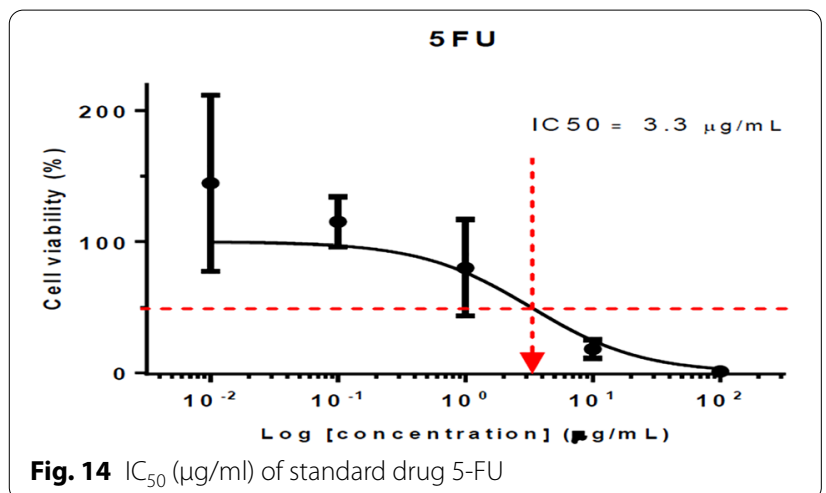

\section{Conclusion}

All the compounds were synthesized according to synthetic scheme under appropriate experimental conditions and analysed by elemental analysis, IR, mass, and ${ }^{1} \mathrm{H} /{ }^{13} \mathrm{CNMR}$. The pharmacological potential was evaluated to study the effect of different substituents on antimicrobial, antioxidant and anti-urease activities. From the outcomes of the pharmacological studies it can be concluded that the substitution of tri-methoxy $\left(\mathbf{T}_{5}\right)$ group increases the antibacterial activity whereas introduction of nitro $\left(\mathbf{T}_{17}\right)$ group at $p$-position enhances the antifungal activity. The introduction of aldehyde $\left(\mathbf{T}_{2}\right)$ and methyl $\left(\mathbf{T}_{3}\right)$ at $p$-position of aromatic ring may increase the anti-urease as well as antioxidant activities. The substitution of $p$-aldehyde $\left(\mathbf{T}_{2}\right)$ and $o$-hydroxy $\left(\mathbf{T}_{7}\right)$ groups on the aromatic ring may enhance the anticancer activity against HCT116 cell line.

\begin{abstract}
Abbreviations
IR: Infrared; NMR: Nuclear magnetic resonance; BS: Bacillus subtilis; PA: Pseudomonas aeruginosa; EC: Escherichia coli; CA: Candida albicans; AN: Aspergillus niger; DPPH: 2,2-Diphenyl-1-picryl-hydrazyl-hydrate; MCF-7: Michigan Cancer Foundation-7; HCT116: Human colon cancer cell line; 5-FU: Fluorouracil; MIC: Minimum inhibitory concentration; Cipro: Ciprofloxacin; Amo: Amoxycillin; Flu: Fluconazole; $I_{50}$ : Half maximal inhibitory concentration/median inhibitory concentration; SAR: Structure activity relationship; DNA: Deoxyribose nucleic acid; TLC: Thin layer chromatography; ATR: Attenuated total reflection; DMSO$\mathrm{d}_{6}$ : Di-methyl sulfoxide; ELISA: Enzyme-Linked Immuno Sorbent Assay.
\end{abstract}

\section{Acknowledgements}

The authors are thankful to Head, Department of Pharmaceutical Sciences, Maharshi Dayanand University, Rohtak, for providing necessary facilities to carry out this research work.

\section{Authors' contributions}

Authors MK, ST, BN and SK have designed synthesized and carried out the antimicrobial, antioxidant, anti-urease activities and KR, SML, SAAS and VM have carried out the spectral analysis, interpretation and anticancer evaluation of synthesized compounds. All authors read and approved the final manuscript.

\section{Funding}

Not applicable.

\section{Availability of data and materials}

The datasets used and/or analysed during the current study are available from the corresponding author on reasonable request.
Ethics approval and consent to participate Not applicable.

\section{Competing interests}

The authors declare that they have no competing interests.

\section{Author details}

${ }^{1}$ Faculty of Pharmaceutical Sciences, Maharshi Dayanand University, Rohtak 124001, India. ${ }^{2}$ Faculty of Pharmacy, Universiti Teknologi MARA (UiTM), 42300 Bandar Puncak Alam, Selangor Darul Ehsan, Malaysia. ${ }^{3}$ Collaborative Drug Discovery Research (CDDR) Group, Pharmaceutical Life Sciences Community of Research, Universiti Teknologi MARA (UiTM), 40450 Shah Alam, Selangor Darul Ehsan, Malaysia. ${ }^{4}$ Atta-Ur-Rahman Institute for Natural Products Discovery (AuRIns), Universiti Teknologi MARA (UiTM), Puncak Alam Campus, 42300 Bandar Puncak Alam, Selangor Darul Ehsan, Malaysia. ${ }^{5}$ Department of Pharmacology and Toxicology, College of Pharmacy, Qassim University, Buraidah 51452, Kingdom of Saudi Arabia.

Received: 21 January 2020 Accepted: 11 June 2020

Published online: 21 January 2021

\section{References}

1. Antunes MM, Amarante TR, Valente AA, Almeida Paz FA, Goncalves IS, Pillinger M (2018) A linear trinuclear oxidodiperoxido-molybdenum (vi) complex with single triazole bridges: catalytic activity in epoxidation, alcoholysis, and acetalization reactions. ChemCatChem 10(13):2782-2791

2. Jacob JH, Irshaid Fl, Al-Soud YA, Al-Balushi AM, Al-Arqan HR (2013) Synthesis, characterization and evaluation of antibacterial activity of six novel 1,2,4-triazole derivatives against standard and medical bacteria. Adv Stud Biol 5(6):303-318

3. Asif M (2014) A brief review on antitubercular activity of pharmacological active some triazole analogues. Glob J Res Rev 1(3):51-58

4. Potts KT (1961) The chemistry of 1,2,4-triazoles. Chem Rev 61(2):87-127

5. Shalini K, Kumar N, Drabu S, Sharma PK (2011) Advances in synthetic approach to and antifungal activity of triazoles. Beilstein J Org Chem 7(1):668-677

6. Bonandi E, Christodoulou MS, Fumagalli G, Perdicchia D, Rastelli G, Passarella D (2017) The 1,2,3-triazole ring as a bioisostere in medicinal chemistry. Drug Discov Today 22(10):1572-1581

7. Berova M, Zlatev Z (2000) Physiological response and yield of paclobutrazol treated tomato plants (Lycopersicon esculentum Mill.). Plant Growth Regul. 30(2):117

8. Schiller DS, Fung HB (2007) Posaconazole: an extended-spectrum triazole antifungal agent. Clin Ther 29(9):1862-1886

9. Zhou HC, Wang Y (2012) Recent researches in triazole compounds as medicinal drugs. Curr Med Chem 19(2):239-280

10. Wang $B L$, Zhan $Y Z$, Zhang LY, Zhang Y, Zhang X (2016) Synthesis and fungicidal activities of novel 1,2,4-triazole thione derivatives containing 1,2,3-triazole and substituted piperazine moieties. Phosphorus Sulfur Silicon Relat Elem 191(1):1-7

11. Goss PE (1998) Pre-clinical and clinical review of vorozole, a new third generation aromatase inhibitor. Breast Cancer Res Treat 49(1):S59-65

12. Millson D, Tepper S (2000) Migraine pharmacotherapy with oral triptans: a rational approach to clinical management. Expert Opin Pharmacother 1(3):391-404

13. Paeshuyse J, Dallmeier K, Neyts J (2011) Ribavirin for the treatment of chronic hepatitis $C$ virus infection: a review of the proposed mechanisms of action. Curr Opin Virol 1(6):590-598

14. Singhal N, Sharma PK, Dudhe R, Kumar N (2011) Recent advancement of triazole derivatives and their biological significance. J Chem Pharm Res 3(2):126-133

15. Sancak K, Unver Y, Unluer D, Dugdu E, Kor G, Celic F, Birinci E (2012) Synthesis, characterization, and antioxidant activities of new tri-substituted triazoles. Turk J Chem 36:457-466

16. Bektas H, Ceylan S, Demirba N, Alpay-Karaoglu S, Sokmen BB (2013) Antimicrobial and antiurease activities of newly synthesized morpholine derivatives containing an azole nucleus. Med Chem Res 22(8):3629-3639

17. Tahlan S, Kumar S, Kakkar S, Narasimhan B (2019) Benzimidazole scaffolds as promising antiproliferative agents: a review. BMC Chem 13(1):66 
18. Kingsbury KJ, Paul S, Crossley A, Morgan DM (1961) The fatty acid composition of human depot fat. Biochem J 78:541-550

19. Jensen RG, Hagerty MM, McMahon KE (1978) Lipids of human milk and infant formulas: a review. Am J Clin Nutr 31(6):990-1016

20. Gupta AK, Prachand S, Patel A, Jain S (2012) Synthesis of some 4-amino-5(substituted-phenyl)-4H-[1,2,4] triazole-3-thiol derivatives and antifungal activity. Int J Pharm Life Sci 3(7):1848-1857

21. Al-Omar MA, Al-Abdullah ES, Shehata IA, Habib EE, Ibrahim TM, El-Emam AA (2010) Synthesis, antimicrobial and anti-inflammatory activities of novel 5-(1-adamantyl)-4-arylideneamino-3-mercapto-1,2,4-triazoles and related derivatives. Molecules 15:2526-2550

22. El-Serwy WS, Mohamed NA, Abbas EM, Abdel-Rahman RF (2013) Synthesis and anti-inflammatory properties of novel 1,2,4-triazole derivatives. Res Chem Intermed 39(6):2543-2554

23. Plech T, Kapron B, Luszczki JJ, Wujec M, Paneth A, Siwek A, Nowak $G$ (2014) Studies on the anticonvulsant activity and influence on
GABA-ergic neurotransmission of 1,2,4-triazole-3-thione-based compounds. Molecules 19(8):11279-11299

24. Park BK, Kitteringham NR, Maggs JL, Pirmohamed M, Williams DP (2005) The role of metabolic activation in drug-induced hepatotoxicity. Annu Rev Pharmacol Toxicol 45:177-202

25. Asif M (2015) Antivral and antiparasitic activities of various substituted triazole derivatives: a mini review. Chem Int 1(2):71-80

26. El-Sayed R, Khairou KS (2015) Propoxylated fatty thiazole, pyrazole, triazole, and pyrrole derivatives with antimicrobial and surface activity. J Surfactants Deterg 18(4):661-673

27. El-Sayed R (2006) Synthesis, antibacterial and surface activity of 1,2,4-triazole derivatives. Grasas Aceites 57(2):180-188

\section{Publisher's Note}

Springer Nature remains neutral with regard to jurisdictional claims in published maps and institutional affiliations. 